\title{
Variability of Soil
}

Md. Wasiul Bari

Research Associate, Department of Civil Engineering,

E-mail: Md.Bari@curtin.edu.au

Mohamed A. Shahin $\uparrow$

Associate Professor, Department of Civil Engineering,

E-mail: M.Shahin@curtin.edu.au

$\uparrow$ Corresponding author 


\section{Reliability-Based Semi-Analytical Solution for Ground} Improvement by PVDs Incorporating Inherent (Spatial)

\section{Variability of Soil}

\section{Md. Wasiul Bari and Mohamed A. Shahin}

Abstract: The design of soil consolidation via prefabricated vertical drains (PVDs) has been traditionally carried out deterministically and thus can be misleading due to the ignorance of the uncertainty associated with the inherent (spatial) variation of soil properties. To treat such uncertainty in the design process of soil consolidation by PVDs, stochastic approaches that combine the finite element method with the Monte Carlo technique (FEMC) have been usually used. However, such approaches are complex, computationally intensive and time consuming. In this paper, a simpler reliability-based semi-analytical (RBSA) method is proposed as an alternative tool to the complex FEMC approach for soil consolidation by PVDs, considering soil spatial variability. The RBSA method is found to give similar results to those obtained from the FEMC approach and can thus be used with confidence in practice.

Keywords: Reliability-based design; Soil consolidation; Prefabricated vertical drains; Finite element method; Monte Carlo technique; Soil spatial variability.

\section{Introduction}

Traditionally, to predict soil consolidation by PVDs using available deterministic methods [e.g., 1, 2, 3], it has been usually assumed that the consolidating soil surrounding the 
PVDs is homogeneous. In reality, however, the degree of consolidation achieved via PVDs is strongly dependent on soil properties that are spatially variable in nature, such as soil permeability, $k$, and volume compressibility, $m_{v}$. Consequently, the rate of soil consolidation is difficult to predict deterministically, especially for heterogeneous soil deposits. Therefore, it is crucial to develop more realistic solutions that can accommodate the true nature of the inherent (spatial) variability of soil in the course of design of soil consolidation by PVDs. In recent years, a few attempts have been made to quantify and assess the uncertainty associated with soil consolidation. For example, some studies [i.e., 4, 5, 6] focussed on the impact of soil variability in one dimensional consolidation due to vertical drainage (i.e., no PVDs). A few more studies [i.e., 7, 8] focussed on the uncertainty associated with the measurement errors of soil testing for PVD-improved ground but soil spatial variability has not been explicitly investigated. More recently, Walker and Indraratna [9] proposed an analytical model incorporating a parabolic permeability distribution in the smear zone, and Basu et al. [10] performed a study to include a transition zone of linearly varying permeability between the smear and undisturbed zones with constant permeability. The above solutions, despite of being useful, failed to accommodate the true nature of soil spatial variability in design of ground improvement by PVDs and more alternative realistic solutions are needed. In order to treat soil spatial variability in most geotechnical engineering problems, stochastic computational schemes that combine the finite element method and Monte Carlo technique [e.g., 6, 11, 12] have been often used. Despite the fact that such schemes offer successful solutions, they require a large number of simulations that are computationally intensive and time consuming. In the current study, an alternative simplified reliability-based semi-analytical (RBSA) approach is introduced for design of soil consolidation by PVDs, considering the spatial variations of soil permeability, $k$, and volume compressibility, $m_{v}$. The developed RBSA method is verified by comparing its results with those obtained from the 
complex stochastic 3D finite-element Monte-Carlo (FEMC) approach and the results are found to be in a good agreement. In the sections that follow, the stochastic FEMC approach is demonstrated first followed by detailed description of the alternative RBSA method.

\section{Stochastic finite-element Monte-Carlo approach}

For the purpose of examining the proposed RBSA method which will be discussed later in detail in the following section, a series of stochastic FEMC analyses are performed and their results are used for comparison with the RBSA method. The FEMC approach merges the local average subdivision (LAS) technique [13] and finite element (FE) modelling into a Monte Carlo framework using the following steps:

1. Identify the spatially variable soil properties affecting soil consolidation by PVDs;

2. Create a virtual soil profile that contains random fields of designated soil properties;

3. Incorporate the generated random fields of soil profile into FE modelling; and

4. Repeat Steps 2 and 3 many times using the Monte Carlo technique so that a series of consolidation responses is obtained from which probabilistic solution for soil consolidation can be derived.

The above steps, as well as the numerical procedures, are described below.

\subsection{Identification of significant spatially variable soil properties}

As indicated earlier, spatial variability of several soil properties can affect soil consolidation by PVDs. However, as confirmed by several researchers [e.g., 6, 14], soil permeability, $k$, and volume compressibility, $m_{v}$, are the most significant factors affecting soil consolidation by PVDs. Although the coefficients of permeability in the vertical and 
horizontal directions (i.e., $k_{v}$ and $k_{h}$, respectively) may vary in the ground, the impact of $k_{h}$ is dominant [8]. Consequently, in the current study, only $k_{h}$ and $m_{v}$ are considered to be spatially variables, while the other soil properties are held constant and treated deterministically so as to reduce the superfluous complexity to the problem.

\subsection{Generation of random fields of soil properties}

In this study, the LAS method [13] extracted from the random field theory [15] are used to generate virtual random fields that allow rational random distributions of $k_{h}$ and $m_{v}$, which are then implemented in the FEM modelling. Based on the random field theory, a random field of certain probability distribution of spatially variable soil property can be characterised by the soil property mean value, $\mu$, variance, $\sigma^{2}$ (can also be represented by the standard deviation, $\sigma$, or coefficient of variation, $v$, where $v=\sigma / \mu$ ) and correlation length or scale of fluctuation, $\theta$. The value of $\theta$ describes the limits of spatial continuity and can simply be defined as the distance over which a soil property shows considerable correlation between two spatial points. Therefore, a large value of $\theta$ indicates strong correlation (i.e., uniform soil property field), whereas a small value of $\theta$ implies weak correlation (i.e., erratic soil property field).

In the current study, lognormally distributed random fields are assumed for simulating the spatial variability of $k_{h}$ and $m_{v}$ because this distribution is extensively used in the literature both for $k_{h}$ and $m_{v}[5,6,16]$. To create a random field of soil property $X$, the following process is followed. A correlated local (arithmetic) average of normally distributed random field $G_{X}(i)$ over the domain of the $i$ th element are first generated for 3D grid of soil mass with values of soil property of zero mean, unit variance and scale of fluctuation $\theta_{X}$. The required 
lognormally distributed random field defined by $\mu_{X}$ and $\sigma_{X}$ is then obtained using the

$$
\sigma_{\ln X}=\sqrt{\ln \left(1+\frac{\sigma_{X}^{2}}{\mu_{X}^{2}}\right)}=\sqrt{\ln \left(1+v_{X}^{2}\right)}
$$

Rearranging Eqs. (2) and (3) gives the following inverse relationships for the mean and

$$
\rho(\tau)=\exp \left\{-\sqrt{\left(\frac{2 \tau_{x}}{\theta_{x}}\right)^{2}+\left(\frac{2 \tau_{y}}{\theta_{y}}\right)^{2}+\left(\frac{2 \tau_{z}}{\theta_{z}}\right)^{2}}\right\}
$$

where $\tau_{x}, \tau_{y}$ and $\tau_{z}$ are, respectively, the distances between two points in $x, y$ and $z$ directions; and $\theta_{x}, \theta_{y}$ and $\theta_{z}$ are, respectively, the scales of fluctuation in $x, y$ and $z$ directions. It should be noted that the spatial correlation function in Eq. (6) becomes statistically isotropic when $\theta_{x}=$ 
underlying normally distributed random field (i.e., $\ln X$ ). Details on the estimation of the scale of fluctuation can be found in Lloret-Cabot et al. [18].

\subsection{Finite element modelling incorporating soil spatial variability}

The subsurface profile simulated in the previous step with the specified spatial variation of $k_{h}$ and $m_{v}$ can now be employed as inputs into a FE consolidation modelling of soil improvement by PVDs. In this study, all numerical analyses are carried out using a modified version of the FE computational scheme "Program 8.6" from the book by Smith and Griffiths [19] in which soil consolidation is treated as 3D uncoupled problem solved using implicit time integration with the "theta" method. The authors modified the source code of "Program 8.6" to incorporate the volume compressibility and allow for repetitive MonteCarlo analyses.

To demonstrate the validity of the proposed RBSA method against the FEMC approach, a consolidation problem is considered for comparison implying a unit cell of soil with central cylindrical drain of dimensions $L=1.0 \mathrm{~m}, r_{e}=0.536 \mathrm{~m}, r_{s}=0.197 \mathrm{~m}$ and $r_{w}=0.032 \mathrm{~m}$ (see Fig. 1a). In the FE analyses, the circular influence area of the cylindrical unit cell is transformed to an equivalent square influence area (see Fig. 1b) of a side length $S=\sqrt{\pi r_{e}^{2}}$ (i.e., $S=0.95 \mathrm{~m}$ ). The selection of the equivalent square influence geometry in the FE modelling is convenient because it avoids the unfavourable mesh shape for the LAS method which requires square (or rectangular) elements to accurately compute locally averaged values of $k_{h}$ and $m_{v}$ for each element across the soil mass. For the same reason, a square shaped smear zone of side length $S_{s}=0.35 \mathrm{~m}$ and PVD of a side length $S_{w}=0.05 \mathrm{~m}$ are also employed in the FE modelling.

It is well known that the overall consolidation of PVD-improved ground is governed by the radial (horizontal) flow of water rather than the vertical flow as the drainage length in the 
horizontal direction is much less than that of the vertical direction and $k_{h}$ is often much higher than that of $k_{v}$ [2]. Under this reasoning, soil consolidation due to the horizontal drainage only is considered in the current study. Neglecting the vertical flow in the FE analyses is matched by setting $k_{v}$ to be equal to zero and since the permeability variance is often described without referring to any direction, the two components of $k_{h}$ (i.e., $k_{x}$ and $k_{y}$ ) are assumed to be isotropic (i.e., $k_{x}=k_{y}=k_{h}$ ).

Although the accuracy of the FE solutions increases with the increase of the number of elements in the FE mesh, a trade-off between accuracy and run-time efficiency is necessary. Previous literature includes some recommendations regarding the optimum ratio of the scale of fluctuation to the finite element size. For example, Ching and Phoon [20] stated that this ratio should be $\geq 20$, whereas Harada and Shinozuka [21] pointed out that it should be $\geq 2$. In the current study, a sensitivity analysis on various FE mesh dimensions is conducted and it is found that a discretization of the FE mesh with an element of size $0.05 \mathrm{~m} \times 0.05 \mathrm{~m} \times 0.05 \mathrm{~m}$ gives a reasonable precision and complies with the recommendation given by Harada and Shinozuka [21]. The 3D mesh used consists of 7220 eight node hexahedral elements (see Fig. 1b). The initial condition for the uncoupled analysis (i.e., no displacement degrees of freedom and only pore pressure degrees of freedom) is such that the excess pore pressure at all nodes (except at the nodes of the drain boundary) is set equal to $100 \mathrm{kPa}$, while the excess pore pressure at each node of the drain boundary is set equal to zero.

During the mandrel installation of PVDs, a disturbed zone surrounding the drain (i.e., smear zone) of reduced $k_{h}$ and increased $m_{v}$ is produced. However, soil spatial variability in the smear zone persists [22], albeit no longer fully natural. Under this reasoning, two groups of RBSA models are developed in this study under various assumed ground conditions. In the first group, the spatially variable soil properties are assumed to be continuous over the whole unit cell. However, non-stationary mean for the spatially variable soil properties are used to 
take into account the smear effect. In this case, the random fields of the spatially variable soil properties in the smear zone are generated separately from those of the undisturbed zone; however, this is carried out in such a way that the ratio of soil permeability in the undisturbed zone to the smear zone (i.e., $k_{h} / k_{h}^{\prime}$ ) and the ratio of volume compressibility in the smear zone to the undisturbed zone (i.e., $m_{v}^{\prime} / m_{v}$ ) are held constant. In the second group, it is assumed that the spatially variable soil properties in the smear zone are completely independent of those of the undisturbed zone. In this case, the random fields of the spatially variable soil properties for the undisturbed and smear zones are generated separately with their corresponding dimension and specified random field parameters. The well resistance is another factor that may affect the efficiency of PVD-improved ground, which is caused due to the deformation of the drain (i.e., folding, bending, crimping) and infiltration of fine soil particles through the drain filter. However, the discharge capacity of most available PVDs in the market is relatively high and well resistance can thus be practically ignored [23], which is the case in the presented example herein. It should be noted though that the proposed RBSA method can also take into account the well resistance effect, if needed.

In the current study, $\mu_{k_{h}}$ and $\mu_{m_{v}}$ are taken to be equal to $0.15 \mathrm{~m} /$ year and $1.0 \times 10^{-3} \mathrm{~m}^{2} / \mathrm{kN}$, respectively. The ratio $\mu_{k_{h}} / \mu_{k_{h}^{\prime}}$, which may vary from 2 to 6 as reported by various researchers [e.g., 2, 23], is taken to be equal to 3 . The ratio $\mu_{m_{v}^{\prime}} / \mu_{m_{v}}$ is taken to be 1.2 , which is in accordance with the value reported by Walker [24]. In order to validate the proposed RBSA, it is decided to conduct the study over the following range of $v$ and $\theta$ for $k_{h}$ and $m_{v}$ :

- $v_{k_{h}}$ (for both the smear and undisturbed zones) $=100,200,300(\%)$

- $v_{m_{v}}$ (for both the smear and undisturbed zones) $=10,20,30(\%)$

- $\theta_{k_{h}}=\theta_{m_{v}}=\theta$ (for both the smear and undisturbed zones) $=0.1,0.25,0.5,1.0,5.0,10.0(\mathrm{~m})$ 
It should be noted that the abovementioned selected ranges of $v$ and $\theta$ for $k_{h}$ and $m_{v}$ are typical to those reported in the literature [e.g., 25, 26] and are believed to represent sufficiently the practical values that can establish general trends for the stochastic soil consolidation behaviour. A single generation of the random fields and the subsequent FE analysis is termed "realization". For an individual realization, the degree of consolidation at any certain consolidation time, $t$, is expressed as $U(t)$ and can be calculated with the help of the following expression:

$U(t)=1-\frac{\bar{u}(t)}{u_{0}}$

where $u_{0}$ is the initial pore pressure and $\bar{u}(t)$ is the average pore pressures at any $t$ (calculated by numerically integrating the excess pore pressure across the mesh and dividing by the total mesh volume), of the consolidation process.

\subsection{Repetition of process based on the Monte Carlo technique}

Following the procedures of the Monte Carlo technique, the process of generating random fields of $k_{h}$ and $m_{v}$ and the subsequent FE analysis is repeated numerous times with the same $v$ and $\theta$ until an acceptable accuracy of estimated statistics of $U(t)$ is achieved. It is found that 2000 Monte Carlo simulations are sufficient to yield reliable and reproducible estimates. One single case of FE analysis with 2000 Monte-Carlo simulations typically takes 6 days on an Intel core i5 CPU @ $3.4 \mathrm{GHz}$ computer. The above repetitive process is performed for each combination of the selected $v$ and $\theta$, and the obtained outputs from each realization of the Monte Carlo procedures are collated and statistically analyzed to make a comparative study between the FEMC approach and the proposed RBSA method, as will be seen later. 


\section{Reliability-based semi-analytical model}

242

It is not uncommon that practicing engineers have neither the time nor the resources to perform full scale FEMC simulations of soil consolidation by PVDs including spatially random properties. Therefore, in this study, an approximate, easy to use reliability-based semi-analytical (RBSA) model is introduced from which direct estimates of the probability of achieving certain $U(t)$ can be readily obtained. The development of the RBSA model requires a performance function or a theoretical (deterministic) model as the commencing point to travel through to the reliability (stochastic) solution. Available deterministic analytical solutions for soil consolidation by PVDs are based on the unit cell concept for a single drain, which is also adopted in the RBSA model. It should be noted that the unit cell concept is deemed to be valid for stochastic analysis of PVD-improved ground because it was found in a recent study carried out by the authors using the FEMC approach that the multi-drain behaviour can be well represented by an idealized unit cell analysis, provided that certain factorized statistical parameters, computed by taking into account the size of the unit cell, are used so as to give equivalent solutions to those of the multi-drain. Detailed description of the validity of the unit cell concept for stochastic analyses of PVD-improved ground as compared to the multi-drain solution is beyond the scope of this paper and can be found elsewhere [see, 27].

In the current study, the commonly used radial consolidation equation of Hansbo [2] is used as the commencing point towards the RBSA model. This equation considers the unit cell concept and has gained a wide acceptance in practical application. The solution is based on the equal strain hypothesis and can estimate the degree of consolidation due to the horizontal drainage, $U_{h}(t)$, at any time, $t$, as given in the following equation (note that as the vertical flow 
265 is ignored in the FE solution, $U_{h}(t)$ from Hansbo's theory will be equal to $U(t)$ of the FE

266 analysis and can be simply denoted as $U(t))$.

267

$U(t)=1-\exp \left(-\frac{2 k_{h} t}{m_{v} \gamma_{w} r_{e}^{2} \alpha}\right)$

268

$\alpha=F_{n}+F_{s}+F_{r}$

269

270

271

272

273

274

275

276

277

278

279

280

281 zone that is denoted earlier as $m_{v}^{\prime}$. The ignorance of the increased $m_{v}$ in the smear zone may

where $F_{n}, F_{s}$ and $F_{r}$ are the drain spacing factor, smear factor and well-resistance factor, respectively, and can be determined by:

$F_{n}=\frac{n^{2}}{\left(n^{2}-1\right)}\left[\ln (n)-\frac{3}{4}+\frac{1}{n^{2}}-\frac{1}{4 n^{2}}\right] \approx \ln (n)-\frac{3}{4}$

$F_{s}=\left(\frac{k_{h}}{k_{h}^{\prime}}-1\right) \ln \left(\frac{r_{s}}{r_{w}}\right)=\left(\frac{k_{h}}{k_{h}^{\prime}}-1\right) \ln (s)$

$F_{r}=\pi z(2 L-z) \frac{k_{h}}{q_{w}}$

where $\gamma_{w}$ is the unit weight of water; $r_{e}$ is the radius of the equivalent soil cylinder with impermeable perimeter (or the radius of zone of influence); $t$ is the consolidation time; $\alpha$ is a group parameter representing the smear effect and geometry of the PVD system; $n=r_{e} / r_{w}$ is the drain spacing ratio ( $r_{w}$ is the equivalent radius of the drain); $s=r_{s} / r_{w}$ is the smear ratio $\left(r_{s}\right.$ is the radius of the smear zone); $k_{h}^{\prime}$ is the horizontal permeability of the smear zone; $q_{w}$ is the vertical discharge capacity of the drain; $L$ is the maximum vertical drainage distance; and $z$ is the depth from the top of the consolidating layer. All parameters shown in Eqs. (8-12) are illustrated in Fig. 1.

It is mentioned earlier that the installation procedure of PVDs not only reduces $k_{h}$ but also increases $m_{v}$ within the smear zone, leading to different volume compressibility in the smear lead to a lack of precision in the analysis. However, $\alpha$ parameter in Eq. (8) proposed by 
Hansbo [2] disregards $m_{v}^{\prime}$. In an effort to rectify this situation, Walker [24] introduced a new

parameter termed as the smear zone volume compressibility parameter, $\alpha_{m_{v}}$, is included in

Eq. (8) to take into account $m_{v}^{\prime}$. For a single smear zone with constant increased volume compressibility, $\alpha_{m_{v}}$ is given by Walker [24] as follows:

$$
\alpha_{m_{v}}=\frac{n^{2}-s^{2}}{n^{2}-1}+\frac{s^{2}-1}{n^{2}-1} \frac{m_{v}^{\prime}}{m_{v}}
$$

By including $\alpha_{m_{v}}$ into Eq. (8), a modified form of this equation is thus:

$$
U(t)=1-\exp \left(-\frac{2 k_{h} t}{m_{v} \gamma_{w} r_{e}^{2} \alpha \alpha_{m_{v}}}\right)
$$

If the changes of $m_{v}$ in the smear zone are not considered, then $\alpha_{m_{v}}$ in Eq. (14) will be equal

to 1.0. That is, Eq. (14) will return back to its original form of Hansbo's [2] formula presented in Eq. (8). Since $k_{h}$ and $m_{v}$ are the only random variables, rearranging Eq. (14) and defining $\ln [1 /\{1-U(t)\}]$ as $U^{*}(t)$ gives:

$$
U *(t)=\frac{2 t}{r^{2} \gamma_{w}} \frac{k_{h}}{m_{v} \alpha \alpha_{m_{v}}}
$$

$$
\text { The above conversion of Eq. (14) to Eq. (15) is necessary as it simplifies the process of }
$$
obtaining a closed form solution for the mean and variance of the degree of consolidation function $U^{*}(t)$ directly from the statistically defined input data (i.e., mean and variance) of $k_{h}$ and $m_{v}$.

$$
\text { The reliability-based solution requires determination of a reasonable probability }
$$
distribution of $U^{*}(t)$, once found, the statistical parameters of the distribution of $U^{*}(t)$ can be estimated. In this regard, simple semi-analytical relationships are derived to aid the designer in estimating the statistical parameters of the distribution of $U^{*}(t)$ directly from the random field parameters. This involves considering an approximate model where the geometric 
averages of $k_{h}$ and $m_{v}$ (i.e., $\bar{k}_{h}$ and $\bar{m}_{v}$, respectively) over the influence zone surrounding the PVD are used in Eq. (15). If the consolidating soil domain surrounding the PVD is termed $D$ and discretized into an assembly of non-overlapping rectangular (or square) elements, then $\bar{k}_{h}$ and $\bar{m}_{v}$ over $D$ can be defined as:

$$
\bar{k}_{h}=\left[\prod_{i=1}^{j} k_{h_{i}}\right]^{1 / j}=\exp \left[\frac{1}{j} \sum_{i=1}^{j} \ln k_{h_{i}}\right]=\exp \left\{\mu_{\ln k_{h}}+\sigma_{\ln k_{h}} \bar{G}_{k_{h}}(D)\right\}
$$

$$
\bar{m}_{v}=\left[\prod_{i=1}^{j} m_{v_{i}}\right]^{1 / j}=\exp \left[\frac{1}{j} \sum_{i=1}^{j} \ln m_{v_{i}}\right]=\exp \left\{\mu_{\ln m_{v}}+\sigma_{\ln m_{v}} \bar{G}_{m_{v}}(D)\right\}
$$

where $i=1,2, \ldots, j$ represents the element number, $\bar{G}_{k_{h}}(D)$ and $\bar{G}_{m_{v}}(D)$ are the arithmetic averages of $G_{k_{h}}(i)$ and $G_{m_{v}}(i)$, respectively, over the domain $D$. It should be noted that $k_{h}$ and $m_{v}$ are assumed to be uncorrelated in the proposed RBSA model, which is due to the lack of data available in the literature to identify the degree and nature of the cross-correlation between $k$ and $m_{v}$. For the problem of one dimensional consolidation, Freeze [5] reported that non-zero cross-correlation between $k$ and $m_{v}$ has a minor impact on the stochastic results of soil consolidation. Prior to finding the distribution and statistical parameters of $U^{*}(t)$, a brief discussion in regard to the underlying equivalent normally distributed mean and variance of the lognormally distributed soil property $X$ (i.e., $\mu_{\ln \bar{X}}$ and $\sigma_{\ln \bar{X}}^{2}$ ) is essential, as follows.

As mentioned earlier, the overall behaviour of PVD system is not governed by the soil properties at discrete points but rather by the average soil properties of the soil volume within the soil domain. For example, in a consolidating heterogeneous soil mass, high flow rates in some regions of high $k$ are offset by lower flow rates in other regions of low $k$, meaning that the total flow from the vicinity of PVD is effectively an averaging process. Despite the fact that the input statistics (i.e., $\mu, \sigma$ and $\theta$ ) characterizing the random soil property of interest is defined at the point level, soil properties are rarely measured at a point and most 
engineering measurements concerned with soil properties are performed on samples of some finite volume, thus actually locally averaged over the sample volume. In light of this, the flow of water through spatially variable soil into the drain is essentially a process governed by the locally averaged soil properties. The local averaging is performed on the underlying point distribution (i.e., normal distribution) of the soil property of interest, which will lead to a reduction in the underlying point variance but the underlying mean will not be affected. For the lognormal distribution, however, both the mean and variance will be reduced by the local averaging, as the mean of a lognormal distribution depends on both the mean and variance of the underlying normal distribution. On the basis of the above discussion, the locally averaged mean of the underlying equivalent $\log$-soil property field $(\ln X), \mu_{\ln \bar{X}}$, which is unaltered by the local averaging can be given by:

$\mu_{\ln \bar{X}}=\mu_{\ln X}$

Using Eqs. (2) and (3), $\mu_{\ln \bar{X}}$ can be expressed in terms of the input statistics of $X$, as follows:

$\mu_{\ln \bar{X}}=\mu_{\ln X}=\ln \mu_{X}-\frac{1}{2} \ln \left(1+v_{X}^{2}\right)$

According to the local averaging theory [15], the variance, $\sigma_{\ln \bar{X}}^{2}$, which is affected by the local averaging, is given by:

$$
\sigma_{\ln \bar{X}}^{2}=\gamma(D) \sigma_{\ln X}^{2}
$$

where $\gamma(D)$ is the "variance function" that defines the amount by which the variance is reduced as a result of the local (arithmetic) averaging over a domain $D$ and is a function of the size of the averaging domain and correlation function. The detailed calculation procedure of the variance reduction factor from the correlation function is given in Appendix A. It should be noted that, since the spatial variability of both $k_{h}$ and $m_{v}$ are modelled using 3D random fields and the FEMC results are obtained from 3D FEM analyses, $\gamma(D)$ in this study is also 
calculated using the 3D variance reduction function. By substituting Eq. (3) into Eq. (20), $\sigma_{\ln \bar{X}}^{2}$ can be expressed in terms of the prescribed statistics of $X$, as follows:

$\sigma_{\ln \bar{X}}^{2}=\gamma(D) \ln \left(1+v_{X}^{2}\right)$

For the purpose of comparing the proposed RBSA method with the FEMC approach, two groups of RBSA models are developed. The random soil properties are considered to be continuous over the whole unit cell in the first group, whereas random soil properties of the smear zone in the second group are assumed to be independent of the undisturbed zone. For each group, two RBSA models are developed to comply with the cases of considering both $k_{h}$ and $m_{v}$ as random variables, while only $k_{h}$ is considered to be a random variable in the second case. For convenience, the RBSA models are denoted as G1C1 and G1C2 for the first group, whereas they are denoted as $\mathrm{G} 2 \mathrm{C} 1$ and $\mathrm{G} 2 \mathrm{C} 2$ for the second group. Considering the readership of the paper, only the two most general RBSA models, namely G1C1 and G2C2, are presented in th section below, whereas the other two RBSA models (i.e., G1C2 and G2C2) are presented in Appendix C. To facilitate the use of the RBSA models, an illustrated worked example will follow.

\subsection{G1C1: RBSA model considering $k_{h}$ and $m_{v}$ as continuous random variables over the entire} unit cell

$$
\text { In the development of the RBSA-G1C1 model, it is assumed that both } k_{h} \text { and } m_{v} \text { vary }
$$
spatially in such a way that their second moment structures (variance, covariance, etc.) in the undisturbed and smear zones are identical with respect to the mean (i.e., $v_{k_{k}}=v_{k_{h}^{\prime}}, \theta_{k_{h}}=\theta_{k_{h}^{\prime}}$ and $v_{m_{v}}=v_{m_{v}^{\prime}}, \theta_{m_{v}}=\theta_{m_{v}^{\prime}}$. This means that the variance and covariance structure is assumed to be stationary. However, non-stationary means for $k_{h}$ and $m_{v}$ are used to take into account the smear effect. This is considered because non-stationary correlation structures are uncommon 
377 in geotechnical engineering due to the prohibitive volumes of data required to estimate their

378 parameters. In geotechnical engineering, random-field models are often non-stationary in their

379 mean; however, the variance and covariance structure is generally assumed to be stationary

380 (Fenton and Griffiths 2008). As $k_{h}$ and $m_{v}$ are continuous over the entire soil domain, each

381 point in the unit cell is correlated to each other. Therefore, it can be assumed that $\mu_{k_{h}} / \mu_{k_{h}^{\prime}}$ and

$382 \mu_{m_{v}^{\prime}} / \mu_{m_{v}}$ remain constant in the unit cell. In other words, $\alpha$ and $\alpha_{m_{v}}$ contribute with little or no

383 variability to $U^{*}(t)$. Considering $k_{h}$ and $m_{v}$ as the only random variables and using their

384 geometric averages, Eq. (15) becomes:

385

$U *(t)=C \frac{\bar{k}_{h}}{\bar{m}_{v}}$

where $\bar{k}_{h}$ and $\bar{m}_{v}$ are, respectively, the geometric averages of soil permeability and volume compressibility;

388

$C=\frac{2 t}{r_{e}^{2} \gamma_{w} \alpha \alpha_{m_{v}}}$

389

$\alpha=\ln \left(\frac{n}{s}\right)-\frac{3}{4}+\frac{\mu_{k_{h}}}{\mu_{k_{h}^{\prime}}} \ln (s)+F_{r}^{\prime}$

$390 \quad \alpha_{m_{v}}=\frac{n^{2}-s^{2}}{n^{2}-1}+\frac{s^{2}-1}{n^{2}-1} \frac{\mu_{m_{v}^{\prime}}}{\mu_{m_{v}}}$

391 Since the random variation of well resistance effect is not considered in this study, $F_{r}^{\prime}$ in Eq.

392 (24), which represents the average well resistance effect over the entire drain length, can be

393 estimated as [28]:

$394 \quad F_{r}^{\prime}=\frac{2 \pi L^{2}}{3} \frac{\mu_{k_{h}}}{q_{w}}$

395 Now a reasonable distribution for $U^{*}(t)$ can be found. Since both $k_{h}$ and $m_{v}$ are assumed to be

lognormally distributed, then $\bar{k}_{h}$ and $\bar{m}_{v}$ are also lognormally distributed (based on the central 
397 limit theorem, the geometric average of a random variable tends to have a lognormal

398 distribution), and therefore $U^{*}(t)$ will be lognormally distributed. In such a case, taking the 399 logarithm of Eq. (22) yields:

$400 \quad \ln U *(t)=\ln C+\ln \bar{k}_{h}-\ln \bar{m}_{v}$

401 To evaluate the probability of achieving a certain $U(t)$, the mean $\mu_{\ln U^{*}(t)}$ and variance $\sigma_{\ln U^{*}(t)}^{2}$

402 of $\ln U^{*}(t)$ need to be estimated. The mean $\mu_{\ln U^{*}(t)}$ of $\ln U^{*}(t)$ can be obtained by taking the

403 expectation of Eq. (27), as follows:

$404 \quad \mu_{\ln U^{*}(t)}=\ln C+\mu_{\ln \bar{k}_{h}}-\mu_{\ln \bar{m}_{v}}$

405 Assuming no cross-correlation between $k_{h}$ and $m_{v}$, the variance $\sigma_{\ln U^{*}(t)}^{2}$ of $\ln U^{*}(t)$ can be

406 simply estimated, as follows:

$407 \quad \sigma_{\ln U^{*}(t)}^{2}=\sigma_{\ln \bar{k}_{h}}^{2}+\sigma_{\ln \bar{w}_{v}}^{2}$

408 The four unknown parameters: $\mu_{\ln \bar{k}_{h}}, \mu_{\ln \bar{m}_{v}}, \sigma_{\ln \bar{k}_{h}}^{2}$ and $\sigma_{\ln \bar{m}_{v}}^{2}$ in Eqs. (28) and (29) are now need 409 to be expressed in terms of the known statistical input parameters of $k_{h}$ and $m_{v}$. With reference 410 to Eq. (19), the following expressions of $\mu_{\ln \bar{k}_{h}}$ and $\mu_{\ln \bar{m}_{v}}$ are obtained:

411

$\mu_{\ln \bar{k}_{h}}=\mu_{\ln k_{h}}=\ln \mu_{k_{h}}-\frac{1}{2} \ln \left(1+v_{k_{h}}^{2}\right)$

412

$\mu_{\ln \bar{m}_{v}}=\mu_{\ln m_{v}}=\ln \mu_{m_{v}}-\frac{1}{2} \ln \left(1+v_{m_{v}}^{2}\right)$

413 With reference to Eq. (21), $\sigma_{\ln \bar{k}_{h}}^{2}$ and $\sigma_{\ln \bar{m}_{v}}^{2}$ can then be expressed with the specified statistical 414 parameters of $k_{h}$ and $m_{v}$, as follows:

415

$\sigma_{\ln \bar{k}_{h}}^{2}=\gamma(D)_{k_{h}}\left(\ln \left(1+v_{k_{h}}^{2}\right)\right)$

416

$\sigma_{\ln \bar{m}_{v}}^{2}=\gamma(D)_{m_{v}}\left(\ln \left(1+v_{m_{v}}^{2}\right)\right)$ 
where $\gamma(D)_{k_{h}}$ and $\gamma(D)_{m_{v}}$ are the variance reduction factors for $k_{h}$ and $m_{v}$, respectively. As the inherent spatial variability of both $k_{h}$ and $m_{v}$ is pertinent over the whole unit cell, the entire soil domain, $D$, is used for estimating $\gamma(D)_{k_{h}}$ and $\gamma(D)_{m_{v}}$.

Now $\mu_{\ln U^{*}(t)}$ and $\sigma_{\ln U^{*}(t)}^{2}$ can be evaluated by substituting $\mu_{\ln \bar{k}_{h}}$ and $\mu_{\ln \bar{m}_{v}}$ in Eq. (28), and $\sigma_{\ln \bar{k}_{h}}^{2}$ and $\sigma_{\ln \bar{m}_{v}}^{2}$ in Eq. (29), as follows:

$\mu_{\ln U^{*}(t)}=\ln C+\left[\ln \mu_{k_{h}}-\frac{1}{2} \ln \left(1+v_{k_{h}}^{2}\right)\right]-\left[\ln \mu_{m_{v}}-\frac{1}{2} \ln \left(1+v_{m_{v}}^{2}\right)\right]$

$\sigma_{\ln U^{*}(t)}^{2}=\gamma(D)_{k_{h}}\left(\ln \left(1+v_{k_{h}}^{2}\right)\right)+\gamma(D)_{m_{v}}\left(\ln \left(1+v_{m_{v}}^{2}\right)\right)$

Using the developed semi-analytical relationships shown in Eqs. (34) and (35), the procedure for evaluating $\mu_{\ln U^{*}(t)}$ and $\sigma_{\ln U^{*}(t)}^{2}$ can then be summarized as follows:

1. Determine the mean, standard deviation and scale of fluctuation of $k_{h}$ and $m_{v}$ (i.e., $\mu_{k_{h}}$, $\sigma_{k_{h}}$ and $\theta_{k_{h}} ;$ and $\mu_{m_{v}}, \sigma_{m_{v}}$ and $\left.\theta_{m_{v}}\right)$

2. Calculate $v_{k_{h}}=\sigma_{k_{h}} / \mu_{k_{h}}$ and $v_{m_{v}}=\sigma_{m_{v}} / \mu_{m_{v}}$;

3. Evaluate all constant parameters involved in the RBSA method (i.e., $\alpha, \alpha_{m_{v}}, C, \gamma(D)_{k_{h}}$ and $\left.\gamma(D)_{m_{v}}\right) ;$ and

4. Estimate $\mu_{\ln U^{*}(t)}$ and $\sigma_{\ln U^{*}(t)}^{2}$ by substituting $C, \mu_{k_{h}}, \mu_{m_{v}}, v_{k_{h}}$ and $v_{m_{v}}$ in Eq. (34), and $\gamma(D)_{k_{h}}, \gamma(D)_{m_{v}}, v_{k_{h}}$ and $v_{m_{v}}$ in Eq. (35).

\subsection{G2C1: RBSA model considering $k_{h}, k_{h}^{\prime}, m_{v}$ and $m_{v}^{\prime}$ as independent random variables}

As $k_{h}, k_{h}^{\prime}, m_{v}$ and $m_{v}^{\prime}$ are independent random variables, $\alpha$ and $\alpha_{m_{v}}$ are no longer constant parameters. Eq. (15) is therefore becomes: 
$U *(t)=C \frac{\bar{k}_{h}}{\bar{m}_{v} \bar{\alpha} \bar{\alpha}_{m_{v}}}$

439 where

$440 \quad C=\frac{2 t}{r_{e}^{2} \gamma_{w}}$

$441 \bar{\alpha}$ and $\bar{\alpha}_{m_{v}}$ are, respectively, the equivalent $\alpha$ and $\alpha_{m_{v}}$ parameters of the spatially variable soil 442 and can be expressed by the following equations:

443

$\bar{\alpha}=\ln \left(\frac{n}{s}\right)-\frac{3}{4}+\frac{\bar{k}_{h}}{\bar{k}_{h}^{\prime}} \ln (s)+F_{r}^{\prime}$

$444 \quad \bar{\alpha}_{m_{v}}=\frac{n^{2}-s^{2}}{n^{2}-1}+\frac{s^{2}-1}{n^{2}-1} \frac{\bar{m}_{v}}{\bar{m}_{v}^{\prime}}$

445 Assuming that: $\ln \left(\frac{n}{s}\right)-\frac{3}{4}+F_{r}^{\prime}=a ; \ln (s)=b$ and $\frac{\bar{k}_{h}}{\bar{k}_{h}^{\prime}}=W$, Eq. (38) becomes:

446

$\bar{\alpha}=a+b W$

447 Similarly, by assuming $\frac{n^{2}-s^{2}}{n^{2}-1}=g ; \frac{s^{2}-1}{n^{2}-1}=h$ and $\frac{\bar{m}_{v}}{\bar{m}_{v}^{\prime}}=V$, Eq. (39) becomes:

448

$\bar{\alpha}_{m_{v}}=g+h V$

449 The parameters $\alpha$ and $\alpha_{m_{v}}$ are respectively the function of permeability and volume

450

compressibility. Therefore, $\bar{\alpha}$ and $\bar{\alpha}_{m_{v}}$, and in turn $U^{*}(t)$ will also be approximately

451

lognormally distributed. In such a case, the mean $\mu_{\ln U^{*}(t)}$ of $\ln U^{*}(t)$ can be obtained by taking

452

logarithm and subsequent expectation of Eq. (36):

453

$\mu_{\ln U^{*}(t)}=\ln C+\mu_{\ln \bar{k}_{h}}-\mu_{\ln \bar{m}_{v}}-\mu_{\ln \bar{\alpha}}-\mu_{\ln \bar{\alpha}_{m_{v}}}$

454 The variance of $k_{h}, k_{h}^{\prime}, m_{v}$ and $m_{v}^{\prime}$ contribute to the variance of $\ln U^{*}(t)$. As $\bar{\alpha}$ and $\bar{\alpha}_{m_{v}}$

455 involve $k_{h}, k_{h}^{\prime}, m_{v}$ and $m_{v}^{\prime}$, then by assuming no cross-correlation between any of the random

456 variables, the variance $\sigma_{\ln U^{*}(t)}^{2}$ of $\ln U^{*}(t)$ is thus: 
458 In order to obtain $\mu_{\ln U^{*}(t)}$ and $\sigma_{\ln U^{*}(t)}^{2}$ in Eqs. (42) and (43) above, the six unknown

459 parameters $\mu_{\ln \bar{k}_{h}}, \mu_{\ln \bar{m}_{v}}, \mu_{\ln \bar{\alpha}}, \mu_{\ln \bar{\alpha}_{m_{v}}}, \sigma_{\ln \bar{\alpha}}^{2}$ and $\sigma_{\ln \bar{\alpha}_{m_{v}}}^{2}$ must be obtained in terms of the

460 known statistical input parameters of $k_{h}, k_{h}^{\prime}, m_{v}$ and $m_{v}^{\prime}$. The formulations of all unknown

461 parameters are presented in Appendix B, as they are large enough not to be included in the

462 main text so as to avoid any possible disruption to the readership of the paper. At the end of

463 Appendix B, a procedure for calculating these unknown parameters is summarised from

464 which $\mu_{\ln U^{*}(t)}$ and $\sigma_{\ln U^{*}(t)}^{2}$ can be estimated by substituting them in Eqs. (42) and (43).

465 Having established with reasonable accuracy the distribution parameters of $\ln U^{*}(t)$ for

466 the RBSA method, the probabilities of achieving a target degree of consolidation at any

467 specified time, $U_{s}(t)$, can be obtained from the following lognormal probability distribution

468 transformation:

469

$P\left[U *(t) \geq U_{s} *(t)\right]=1-\Phi\left(\frac{\ln U_{s} *(t)-\mu_{\ln U^{*}(t)}}{\sigma_{\ln U^{*}(t)}}\right)$

where: $P[]=$. probability of its argument, $\Phi($.$) is the standard normal cumulative distribution$

471 function and $U_{s}^{*}(t)$ is the target $U^{*}(t)$ that needs to be achieved. Since $U^{*}(t)$ is a

472 monotonically increasing function of $U(t)$, the following equation holds [29]:

$473 \quad P\left[U *(t) \geq U_{s}^{*}(t)\right]=P\left[U(t) \geq U_{s}(t)\right]$

474 Assuming the target degree of consolidation is $90 \%$ (i.e., $U_{s}(t)=0.9$ ) and denoting it as $U_{90}$,

475 the probability of achieving $U_{90}$ at any time, $t$, can be estimated as follows:

476

$$
P\left[U(t) \geq U_{90}\right]=P\left[U^{*}(t) \geq 2.3026\right]=1-\Phi\left(\frac{\ln 2.3026-\mu_{\ln U^{*}(t)}}{\sigma_{\ln U^{*}(t)}}\right)
$$


477 Note that when $U_{s}(t)=U_{90}=0.9$, then $U_{s}^{*}(t)=\ln [1 /(1-0.9)]=2.3026$. In the following

478

479

480

481

482

483

484

485

486

487

488

489

490

491

492

493

494

495

496

497

498

499

500

501 section, detailed comparison between the results obtained from the stochastic FEMC approach and proposed RBSA method is presented and discussed.

\section{Comparison between finite-element Monte-Carlo approach and reliability-} based semi-analytical method

In this section, a comparison between the proposed RBSA method and FEMC approach is demonstrated through an illustrative worked example. For brevity and because of the good agreement between the results of the four proposed RBSA models (i.e., G1C1, G1C2, G2C1 and $\mathrm{G} 2 \mathrm{C} 2$ ) and their corresponding FEMC solutions, only $\mathrm{G} 1 \mathrm{C} 1$ and $\mathrm{G} 2 \mathrm{C} 1$ models are presented herein. It is to be reminded that $k_{h}$ and $m_{v}$ in $\mathrm{G} 1 \mathrm{C} 1$ model are considered as continuous random variables over the entire unit cell, whereas in $\mathrm{G} 2 \mathrm{C} 1$ model $k_{h}$ and $m_{v}$ in the smear and undisturbed zones are considered to be independent random variables. Prior to comparison, the rationality of the assumption of lognormal distribution for $U^{*}(t)$ under various assumed ground conditions is assessed through the frequency density plot of $U^{*}(t)$ on the basis of 2000 realizations for each combination of the variability parameters $v$ and $\theta$ for the spatially variable soil properties at several different consolidation time. The chi-square goodness-of-fit tests for all cases are performed and yielded $p$-values between $0.15-0.96$. Such high $p$-values indicate that there is a very little evidence in the simulated $U^{*}(t)$ sample against the null hypothesis of the assumed lognormal distribution. By accepting the lognormal distribution, all subsequent statistics of the underlying normally distributed $\ln U *(t)$ are estimated by the method of moments from the suite of 2000 realizations using the following transformations:

$\mu_{\ln U^{*}(t)}=\frac{1}{n_{\text {sim }}} \sum_{i=1}^{n_{\text {sin }}} \ln U *_{i}(t)$ 
$\sigma_{\ln U^{*}(t)}=\sqrt{\frac{1}{n_{\text {sim }}-1} \sum_{i=1}^{n_{\text {sim }}}\left[\ln U^{*}{ }_{i}(t)-\mu_{\ln U^{*}(t)}\right]^{2}}$

503 where: $U^{*}(t)$ is the $U^{*}(t)$ from the $i$ th realization $\left(i=1,2,3, \ldots, n_{\text {sim }}\right)$ and $n_{\text {sim }}$ is the total

504 number of realizations (i.e., 2000).

505

4.1 FEMC approach versus RBSA-G1C1 model

507

The illustrative example used for comparison between the FEMC approach and RBSA-

G1C1 model involves the same unit cell consolidation problem illustrated earlier (i.e., $L=$

$1.0 \mathrm{~m}, r_{w}=0.032 \mathrm{~m}, r_{e}=0.536 \mathrm{~m}, r_{s}=0.197 \mathrm{~m}, n=16.75$ and $\left.s=6.156\right)$. The spatial variability

of $k_{h}$ and $m_{v}$ is assumed to have $\mu_{k_{h}}=0.15 \mathrm{~m} /$ year, $k_{h} / k_{h}^{\prime}=3.0, \mu_{m_{v}}=1.0 \times 10^{-3} \mathrm{~m}^{2} / \mathrm{kN}, m_{v}^{\prime} / m_{v}$

512

$=1.2, v_{k_{h}}=200 \%, v_{m_{v}}=20 \%$ and $\theta_{k_{h}}=\theta_{m_{v}}=1.0 \mathrm{~m}$. Armed with the above information,

513

$\mu_{\ln U^{*}(t)}$ (see Eq. (34)) and $\sigma_{\ln U^{*}(t)}^{2}$ (see Eq. (35)) are calculated by following the steps

514

described earlier in developing the RBSA-G1C1 model, as explained below.

method can be calculated using the following equations:

$\alpha=\ln (16.75)-075+(3-1) \times \ln (6.156)=5.703$

518

$\alpha_{m_{v}}=\frac{16.75^{2}-6.156^{2}}{16.75^{2}-1}+\frac{6.156^{2}-1}{16.75^{2}-1} \times 1.2=1.026$

If the probability of achieving $90 \%$ consolidation is to be determined at 0.75 year, then the parameter $C$ will be:

521

$$
C=\frac{2 \times 0.75}{0.536^{2} \times 9.8 \times 5.703 \times 1.026}=0.091 \mathrm{~m} \text { year } / \mathrm{kN}
$$

522

Now using the algorithm presented in Appendix A, the variance reduction factor for $k_{h}$ and $m_{v}$

523 is given by: 
$\gamma(D)_{k_{h}}=\gamma(D)_{m_{v}}=\gamma(S, S, L)=0.312$

Substituting the given $\mu, v$ and the calculated constant parameters in Eqs. (34) and (35) yield:

$\mu_{\ln U^{*}(t)}=\ln (0.091)+\left[\ln 0.15-0.5 \ln \left(1+2.0^{2}\right)\right]-\left[\ln 1.0 \times 10^{-3}-0.5 \ln \left(1+0.2^{2}\right)\right]=1.83$

$\sigma_{\ln U^{*}(t)}^{2}=0.312 \times \ln \left(1+2.0^{2}\right)+0.312 \times \ln \left(1+0.2^{2}\right)=0.514$, therefore, $\sigma_{\ln U^{*}(t)}=0.717$

Using the computed values of $\mu_{\ln U^{*}(t)}$ and $\sigma_{\ln U^{*}(t)}$ in Eq. (46), the probability of achieving $90 \%$ consolidation from the RBSA-G2C1 model can be computed as follows:

$$
P\left[U(t=0.75) \geq U_{90}\right]=1-\Phi\left(\frac{\ln (2.3026)-1.83}{0.717}\right)=0.92
$$

The FEMC approach of the above problem yields $P\left[U(t=0.75) \geq U_{90}\right]=0.94$, thus demonstrating an excellent agreement between the FEMC approach and proposed RBSAG2C1 method. Following the above procedure, $\mu_{\ln U^{*}}, \sigma_{\ln U^{*}}$ and $P\left[U \geq U_{90}\right]$ at each time step over each combinations of the spatial variability parameters are evaluated for both solution approaches and the results are compared in Figs. 2-3. It should be noted that, for brevity, the results of only a few tests are presented.

The agreement between $\mu_{\ln U^{*}}$ and $\sigma_{\ln U^{*}}$ derived from the FEMC simulation and predicted by the RBSA-G1C1 model is examined in Fig. 2. The influence of $v$ on $\mu_{\ln U^{*}}$ is illustrated in Fig. 2(a) for a constant $\theta=0.5 \mathrm{~m}$. It can be seen that, in general, the predicted values of $\mu_{\ln U^{*}}$ obtained from the RBSA model and the FEMC approach match exceptionally well. In both methods, the estimated $\mu_{\ln U^{*}}$ decreases with the increase of $v$, as expected. The relationships between the estimated $\mu_{\ln U^{*}}$ versus the consolidation time, $t$, for various $\theta$ at constant $v_{k_{h}}=200 \%$ and $v_{m_{v}}=20 \%$ are shown in Fig. 2(b). It can be seen that the results obtained from both the FEMC approach and RBSA-G1C1 model are almost identical. In each solution method, even though the results for various $\theta$ are drawn in the plot, they are embodied into a single curve, implying that the obtained results at different $\theta$ are very close and cannot be 
distinguished. The virtually identical curves for all $\theta$ obtained from each method of analysis demonstrate that $\mu_{\ln U^{*}}$ is largely independent of $\theta$. This is expected as in principle $\theta$ does not affect the local average mean of the normally distributed process.

The effect of $v$ on $\sigma_{\ln U^{*}}$ for a fixed value of $\theta=0.5 \mathrm{~m}$ is shown in Fig. 2(c), which shows that, in general, $\sigma_{\ln U^{*}}$ increases with the increase of $v$ and the agreement between the FEMC approach and RBSA model is very good. The influence of $\theta$ on $\sigma_{\ln U^{*}}$ at constant $v_{k_{h}}=200 \%$ and $v_{m_{v}}=20 \%$ is shown in Fig. 2(d). It can be seen that $\sigma_{\ln U^{*}}$ increases with the increase of $\theta$ for both approaches, and apart from some slight discrepancy at high $\theta \geq 5 \mathrm{~m}$, the agreement between the FEMC approach and RBSA model is reasonable and shows good compliance. This behaviour can be explained by noting that, when $\theta \rightarrow 0$, the simulated soil profile is consisted of an infinite number of independent 'observations', thus there is a decrease in the average variance of the consolidation rate and the averaging process almost perfectly predicts the condition in the unit cell. Conversely, when $\theta$ is large, the average variance of the consolidation rate is also expected to be large due to the decrease in the number of independent 'observations', resulting in less averaging variance reduction within each realization.

The agreement between the FEMC approach and RBSA-G1C1 model is examined in terms of $P\left[U \geq U_{90}\right]$ in Fig. 3. The effect of $v$ on $P\left[U \geq U_{90}\right]$ at a fixed value of $\theta=0.5 \mathrm{~m}$ is shown in Fig. 3(a). It can be seen that the two solutions are in a good agreement despite some slight discrepancy at the earlier stage of consolidation. This may be attributed to the fact that the FEMC approach relies on the free strain concept, while the RBSA method is based on Hansbo's solution of an equal strain assumption. As the probability of achieving a target degree of consolidation of usual interest is greater than 50\%, any discrepancy in this range has a little implication from the practical point of view. In Fig. 3(b), the compliance between the FEMC approach and proposed RBSA method shows a good agreement for various $\theta$ at 
constant $v_{k_{h}}=200 \%$ and $v_{m_{v}}=20 \%$, although a slight discrepancy in $P\left[U \geq U_{90}\right]$ exists when $\theta$ is as small as $0.1 \mathrm{~m}$ (i.e., for erratic soil). It can also be seen that for any $P\left[U \geq U_{90}\right] \geq$ $50 \%$, the RBSA-G1C1 model yields slightly higher (unconservative) estimation of $P[U \geq$ $U_{90}$ ] than that calculated by the FEMC approach when $\theta$ is as low as $0.1 \mathrm{~m}$. On the other hand, $P\left[U \geq U_{90}\right]$ derived from the RBSA-G1C1 model is slightly lower (conservative) than those obtained from the FEMC approach when $\theta$ is as high as $1.0 \mathrm{~m}$.

\subsection{FEMC approach versus RBSA-G2C1 model}

Following the procedure set out in Appendix B, curves for $\mu_{\ln U^{*}}, \sigma_{\ln U^{*}}$ and in turn $P[U \geq$ $U_{90}$ ] with time over some selected combinations of the spatial variability parameters are obtained for RBSA-G2C1 method. The agreement between $\mu_{\ln U^{*},} \sigma_{\ln U^{*}}$ and $P\left[U \geq U_{90}\right]$ derived from the FEMC simulation and predicted by the RBSA-G2C1 model are then examined in Figs. 4-9. As mentioned earlier, two independent random fields for $k_{h}$ and $m_{v}$ are generated for the undisturbed and smear zones. For convenience of presentation, the statistical parameters in the smear and undisturbed zones (i.e., $v$ and $\theta$ of $k_{h}$ and $m_{v}$ ) are denoted with appropriate subscripts " $s$ " and " $u$ " depending on whether they are specified for the smear zone or undisturbed zone, where $s$ refers to the smear zone while $u$ refers to the undisturbed zone.

The influence of increasing $v$ on the agreement between the FEMC approach and RBSAG2C1 model in terms of $\mu_{\ln U^{*}}$ at a fixed value of $\theta_{u}=\theta_{s}=1.0 \mathrm{~m}$ is shown in Fig. 4 . It can be seen that, in general, the predicted values of $\mu_{\ln U^{*}}$ obtained from the RBSA model match those obtained from the FEMC approach reasonably well. In both methods, the estimated $\mu_{\ln U^{*}}$ decreases with the increase of $v$, as expected. However, the identical curves for all cases of $v_{u}$ $\left(v_{k_{h}^{\prime}}\right.$ and $v_{m_{v}^{\prime}}$ are fixed at $100 \%$ and $10 \%$, respectively) for both methods in Fig. 4(a) indicate that the effect of increasing $v_{u}$ on $\mu_{\ln U^{*}}$ remains marginal. The effect of $v_{s}$ on $\mu_{\ln U^{*}}$ at fixed 
values of $v_{k_{h}}=100 \%$ and $v_{m_{v}}=10 \%$ is illustrated in Fig. 4(b). It can be seen that, although the agreement between the RBSA-G2C1 model and the FEMC approach is reasonably well, the discrepancy in $\mu_{\ln U^{*}}$ between the two methods becomes higher as $t$ increases. Fig. 4 also demonstrates that the decreasing rate of $\mu_{\ln U^{*}}$ is higher for an increase in $v_{s}$ than $v_{u}$.

The matching of $\mu_{\ln U^{*}}$ obtained from the RBSA-G2C1 model and FEMC approach is examined in Fig. 5 for an increasing $\theta$ at constant values of $v_{k_{k}}=v_{k_{h}^{\prime}}=200 \%$ and $v_{m_{v}}=v_{m_{v}^{\prime}}=$ 20\%. The effect of $\theta_{u}$ on $\mu_{\ln U^{*}}$ for a constant value of $\theta_{s}=0.25 \mathrm{~m}$ is shown Fig. 5(a), whereas the effect of $\theta_{s}$ on $\mu_{\ln U^{*}}$ for a fixed value of $\theta_{u}=0.25 \mathrm{~m}$ is shown in Fig. 5(b). It can be seen that the results obtained from both the FEMC approach and RBSA-G2C1 model are nearly identical. However, a slight discrepancy in $\mu_{\ln U^{*}}$ from the two solution approaches is found when the consolidation time $t$ is as large as 1 year. In each solution method, the single curve for all $\theta$ confirms that $\mu_{\ln U^{*}}$ is independent of $\theta$.

The agreement between the FEMC approach and RBSA-G2C1 model is further illustrated by matching the estimated $\sigma_{\ln U^{*}}$ at different values of $v_{u}$ and $v_{s}$, and at a constant $\theta_{u}$ $=\theta_{s}=1.0 \mathrm{~m}$ (see Fig. 6). It can be seen that, in general, $\sigma_{\ln U^{*}}$ increases with the increase of $v$ and the agreement between the two solution approaches is reasonably well. However, for a certain $v$ at any particular consolidation time $t$, the estimated values of $\sigma_{\ln U^{*}}$ derived from the RBSA-G2C1 model are slightly higher than those obtained from the FEMC approach. The above observation is more accurate for $v_{s}$ (see Fig. 6(b)) than $v_{u}$ (see Fig. 6(a)). The comparison shown in Fig. 6 reveals that $\sigma_{\ln U^{*}}$ is largely insensitive to varying $v_{u}$ and highly sensitive to increasing $v_{s}$.

The effect of $\theta$ derived from the FEMC approach and RBSA-G2C1 model in terms of $\sigma_{\ln U^{*}}$ for fixed values of $v_{k_{k}}=v_{k_{h}^{\prime}}=200 \%$ and $v_{m_{v}}=v_{m_{v}^{\prime}}=20 \%$ is demonstrated in Fig. 7. It can be seen that $\sigma_{\ln U^{*}}$ increases with the increase of $\theta$, and apart from some slight discrepancy at large $\theta$ (i.e., at $\theta \geq 5.0 \mathrm{~m}$ ), the agreement between the two methods is again reasonably well. In 
Fig. 7(a), it can be seen that varying $\theta_{u}\left(\theta_{s}\right.$ is fixed at $\left.0.25 \mathrm{~m}\right)$ has a marginal effect on $\sigma_{\ln U}$, while varying $\theta_{s}$ has a considerable impact on the estimated values of $\sigma_{\ln U^{*}}$ (see Fig. 7(b)). The influence of $v$ on the agreement between the FEMC approach and RBSA-G2C1 model in terms of $P\left[U \geq U_{90}\right]$ at a fixed value of $\theta_{u}=\theta_{s}=1.0 \mathrm{~m}$ is shown in Fig. 8. The effect of increasing $v_{u}$ on $P\left[U \geq U_{90}\right]$ is illustrated in Fig. 8(a). It can be seen that the predicted $P[U$ $\left.\geq U_{90}\right]$ obtained from the proposed RBSA-G2C1 model agrees exceptionally well with those obtained from the FEMC approach for all cases of $v_{u}\left(v_{k_{h}^{\prime}}\right.$ and $v_{m_{v}^{\prime}}$ are fixed at $100 \%$ and $10 \%$, respectively). The virtually identical curves of $P\left[U \geq U_{90}\right]$ in Fig. 8(a) for all $v_{u}$ indicate that $P\left[U \geq U_{90}\right]$ is largely independent of $v_{u}$. Fig. 8(b) illustrates the effect of $v_{s}$ on $P\left[U \geq U_{90}\right]$ at a fixed value of $\theta_{u}=\theta_{s}=1.0 \mathrm{~m}$. Although the overall agreement between the estimated $P[U \geq$ $U_{90}$ ] by the two methods is very good, the caveat, however, is that the RBSA-G2C1 model gives slightly unconservative estimate of $P\left[U \geq U_{90}\right]$ for any $P\left[U \geq U_{90}\right]>50 \%$ and particularly when $v_{k_{h}^{\prime}} \geq 200 \%$ with $v_{m_{v}^{\prime}} \geq 20 \%$. This higher values of predicted $P\left[U \geq U_{90}\right]$ given by the RBSA-G2C1 model is due to the higher predicted $\mu_{\ln U^{*}}$, as shown in Fig. 4(b). Fig. 8 also illustrates that the increasing rate of $P\left[U \geq U_{90}\right]$ with respect to $t$ decreases as $v$ increases and this effect is more pronounced for an increase in $v_{s}$ than $v_{u}$.

Apart from some slight discrepancy particularly when $P\left[U \geq U_{90}\right]$ in the range between $70 \%-90 \%$, the FEMC approach and proposed RBSA-G2C1 model show good agreement for various $\theta_{u}$ (see Fig. 9(a)) and $\theta_{s}$ (see Fig. 9(b)) at constant values of $v_{k}=100 \%$ and $v_{m_{v}}=$ $25 \%$ as illustrated in Fig 9. This discrepancy between the two solutions is expected because of the fact that the variability in $U(t)$ is zero at the beginning of consolidation (i.e., at $t=0.0$ ), and gradually increases with the increase in the consolidation time until it reaches a maximum value at certain intermediate $t$, then decreases with further increase in time until it approaches zero again after the full consolidation is occurred. It can be seen that for any $P\left[U \geq U_{90}\right] \geq$ $50 \%$, the values of $P\left[U \geq U_{90}\right]$ derived from the RBSA-G2C1 model are slightly higher 
(unconservative) than those obtained from the FEMC approach when $\theta$ is as low as $0.25 \mathrm{~m}$, while this trend becomes opposite (conservative) when $\theta$ is as high as $1.0 \mathrm{~m}$. The comparison in Fig. 9(a \& b) reveals that the effect of $\theta_{s}$ on $P\left[U \geq U_{90}\right]$ is more significant than $\theta_{u}$.

The overall conclusion from the above comparison in Figs. 4-9 is that the RBSA-G2C1 model and FEMC approach agree reasonably well despite some discrepancies in the results of $\mu_{\ln U^{*}}, \sigma_{\ln U^{*}}$ and $P\left[U \geq U_{90}\right.$. This is attributed mostly to the empirical adjustment of the RSBA model which is necessary due to the fact that the sum of two lognormally distributed random variables does not have a simple closed form solution. In addition, for both solution methods it is found that the probabilistic behavior of soil consolidation is governed by the spatial variation of the soil properties of the smear zone. This behavior is expected because all expelled water from the PVD must pass through the smear zone.

\section{Discussion}

It is noteworthy that the agreement between the proposed RBSA method and FEMC approach shown above was examined for a consolidation problem of a soil layer having a thickness of $1 \mathrm{~m}$ and isotropic scale of fluctuation. Therefore, to arrive at a general conclusion regarding the validity of the proposed RBSA method compared to the FEMC approach for thicker soil layers of anisotropic correlation structure, the comparison is also tested for a more practical example of a unit cell of thickness of geometry $L=4.25 \mathrm{~m}, r_{e}=0.48 \mathrm{~m}, r_{s}=0.197 \mathrm{~m}$ and $r_{w}=0.032 \mathrm{~m}$, and parameters $\mu_{k_{h}}=0.15 \mathrm{~m} /$ year, $k_{h} / k_{h}^{\prime}=3.0, \mu_{m_{v}}=1.0 \times 10^{-3} \mathrm{~m}^{2} / \mathrm{kN}$ and $m_{v}^{\prime} / m_{v}=1.2$. The 3D FE mesh of such problem consisted of 24,565 eight node hexahedral elements of size $0.05 \mathrm{~m} \times 0.05 \mathrm{~m} \times 0.05 \mathrm{~m}$. The FEMC approach of the problem needed an intensive computational time of 28 days to run 2000 realizations on an Intel core i5 CPU @ 3.4 GHz computer. Therefore, only two FEMC simulation tests, named as FEMC1 and 
FEMC2, are performed considering anisotropic $\theta$. FEMC1 and FEMC2 stand for comparison with RBSA-G1C1 and FEMC2 and RBSA-G2C1 models, respectively. For FEMC1 and its counterpart RBSA-G1C1 model, the random field parameters are assumed to be as follows: $v_{k_{h}}=200 \%, v_{m_{v}}=20 \%, \theta_{x}=\theta_{y}=10.0 \mathrm{~m}$ and $\theta_{z}=1.0 \mathrm{~m}$. For FEMC2 and its counterpart RBSA-G2C1model, the spatial variability of $k_{h}$ and $m_{v}$ is assumed to have $v_{k_{k}}=v_{k_{h}^{\prime}}=200 \%$, $v_{m_{v}}=v_{m_{v}^{\prime}}=20 \%, \theta_{x}=\theta_{y}=10.0 \mathrm{~m}$ and $\theta_{z}=1.0 \mathrm{~m}$. The same $\theta$ for $k_{h}$ and $m_{v}$ for the smear and undisturbed zones are used in this investigation. The computed $\mu_{\ln U^{*}(t)}, \sigma_{\ln U^{*}(t)}$ and $P\left[U \geq U_{90}\right]$ from the two methods are compared in Fig. 10. It can be seen that $\mu_{\ln U^{*}(t)}$ (Fig. 10a), $\sigma_{\ln U^{*}(t)}$ (Fig. 10b) and $P\left[U \geq U_{90}\right.$ (Fig. 10c) obtained from both the FEMC approach and RBSA method are almost identical, implying very good agreement between the two methods. This is due to the fact that the stochastic response of soil consolidation by PVDs is dependent on the ratio of the scale of fluctuation to the dimensions of the influence zone surrounding the PVD, which can be readily taken into account by the use of a variance reduction function. Therefore, the proposed RBSA method can be utilized with confidence as an easy-to-use alternative to the computationally intensive FEMC approach for assessing the reliability of soil consolidation by PVDs in spatially variable soils. Despite the fact that the proposed RBSA method is suitable for hand calculations, it is coded by the authors in FORTRAN to provide a user friendly executable program that can be readily used by practitioners, and the program is available for interested readers upon request.

\section{Conclusions}

$$
\text { Simple reliability-based semi-analytical (RBSA) models for predicting the statistics and }
$$
probability of achieving a target degree of consolidation for PVD-improved ground were 
developed incorporating the inherent (spatial) variability of soils. The performance function of the proposed RBSA models was based on the well-known deterministic equation of Hansbo [2], which considers soil consolidation due to the horizontal drainage only. Under various ground conditions, the proposed RBSA models account for the spatial variability of soil volume compressibility and/or soil permeability, which are considered to be the most significant spatial random variables affecting soil consolidation by PVDs.

The results confirm that there is good agreement between the proposed RBSA method and the finite-element Monte-Carlo (FEMC) approach, implying that the simpler RBSA method negates the need for the computationally intensive and time consuming FEMC technique. The results also indicate that, for given coefficients of variation of soil permeability and volume compressibility, the stochastic response of soil consolidation by PVDs is dependent on the ratio of the scale of fluctuation to the dimensions of the influence zone surrounding the PVD, which can be readily taken into account by the use of a variance reduction function. Therefore, the proposed RBSA model can be confidently employed to assess the reliability of consolidation problems implying arbitrary dimensions.

Despite the success of the proposed RBSA method for design of PVD-improved ground, it has some limitations compared to the FEMC approach which can deal with more general cases and offers the ability to solve problems with less restrictive conditions. For example, the RBSA method does not consider soil consolidation due to the vertical drainage; hence, the computed probability of achieving a target degree of consolidation would be slightly conservative. However, it should be emphasised that, in practice, the contribution of soil consolidation due to the vertical drainage is only a small fraction of the overall soil consolidation and can thus be neglected without significant impact on the design results. In addition, soil permeability and volume compressibility were assumed to be uncorrelated, which again may lead to somewhat conservative solutions. However, it was reported by 
737

$\gamma(X, Y, Z)=\frac{1}{8} \sum_{i=1}^{n_{g}} \omega_{i}\left(1-\psi_{i}\right) \sum_{j=1}^{n_{g}} \omega_{j}\left(1-\psi_{j}\right) \sum_{k=1}^{n_{g}} \omega_{k}\left(1-\psi_{k}\right) \rho\left(\varsigma_{i}, \xi_{j}, \vartheta_{k}\right)$

741 where

$$
\varsigma_{i}=X\left(1+\psi_{i}\right) / 2, \xi_{j}=Y\left(1+\psi_{j}\right) / 2, \vartheta_{k}=Z\left(1+\psi_{k}\right) / 2
$$

743 In which, $\omega_{i}, \vartheta_{i}$, and $n_{g}$ are the weights, Gauss points, and their total number, respectively. 
744 Appendix B. Computation of $\mu_{\ln \bar{k}_{h}}, \mu_{\ln \bar{m}_{v}}, \mu_{\ln \bar{\alpha}}, \mu_{\ln \bar{\alpha}_{m_{v}}}, \sigma_{\ln \bar{\alpha}}^{2}$ and $\sigma_{\ln \bar{\alpha}_{m_{v}}}^{2}$

745

With reference to Eqs. (18) and (19), $\mu_{\ln \bar{k}_{h}}$ and $\mu_{\ln \bar{m}_{v}}$ can be calculated as follows:

747

$\mu_{\ln \bar{k}_{h}}=\mu_{\ln k_{h}}=\ln \mu_{k_{h}}-\frac{1}{2} \ln \left(1+v_{k_{h}}^{2}\right)$

748

$\mu_{\ln \bar{m}_{v}}=\mu_{\ln m_{v}}=\ln \mu_{m_{v}}-\frac{1}{2} \ln \left(1+v_{m_{v}}^{2}\right)$

749

Taking expectation of Eqs. (40) and (41) yield the following equations of the mean of $\bar{\alpha}$ (i.e.,

750

$\mu_{\bar{\alpha}}$ ) and $\bar{\alpha}_{m_{v}}$ (i.e., $\mu_{\bar{\alpha}_{m_{v}}}$ ):

751

$\mu_{\bar{\alpha}}=a+b \mu_{W}$

752

$\mu_{\bar{\alpha}_{m_{v}}}=g+h \mu_{V}$

753 The variance of $\bar{\alpha}$ (i.e., $\sigma_{\bar{\alpha}}^{2}$ ) and $\bar{\alpha}_{m_{v}}$ (i.e., $\mu_{\bar{\alpha}_{m_{v}}}$ ) are thus:

$754 \sigma_{\bar{\alpha}}^{2}=b^{2} \sigma_{W}^{2}$

$755 \quad \sigma_{\bar{\alpha}_{m_{v}}}^{2}=h^{2} \sigma_{V}^{2}$

756 Recalling that, $W=\bar{k}_{h} / \bar{k}_{h}^{\prime}$ and $V=\bar{m}_{v} / \bar{m}_{v}^{\prime}$. Since both $\bar{k}_{h}, \bar{k}_{h}^{\prime}, \bar{m}_{v}$ and $\bar{m}_{v}^{\prime}$ are lognormally

757 distributed, $W$ and $V$ will also be approximately lognormally distributed. According to Eqs.

758 (18) and (19), the following expressions of $\mu_{\ln W}$ and $\mu_{\ln V}$ with the known parameters are

759 derived:

$760 \quad \mu_{\ln W}=\mu_{\ln \bar{k}_{h}}-\mu_{\ln \bar{k}_{h}^{\prime}}=\left(\ln \mu_{k_{h}}-\frac{1}{2} \ln \left(1+v_{k_{h}}^{2}\right)\right)-\left(\ln \mu_{k_{h}^{\prime}}-\frac{1}{2} \ln \left(1+v_{k_{h}^{\prime}}^{2}\right)\right)$

761

$\mu_{\ln V}=\mu_{\ln \bar{m}_{v}^{\prime}}-\mu_{\ln \bar{m}_{v}}=\left(\ln \mu_{m_{v}^{\prime}}-\frac{1}{2} \ln \left(1+v_{m_{v}^{\prime}}^{2}\right)\right)-\left(\ln \mu_{m_{v}}-\frac{1}{2} \ln \left(1+v_{m_{v}}^{2}\right)\right)$ 
Since $k_{h}$ and $k_{h}^{\prime}$ are independent random variables (no correlation between $k_{h}$ and $k_{h}^{\prime}$ ) and

763

764

765

766

767

768

769

770

771

772

773

774

775

776

$777 \quad \mu_{W}=\left(\frac{2 \mu_{\bar{k}_{h}}}{\mu_{\bar{k}_{h}}+\mu_{\bar{k}_{h}^{\prime}}}\right)^{\chi} \exp \left(\mu_{\ln W}+\frac{1}{2} \sigma_{\ln W}^{2}\right)$

$777 \quad \mu_{W}=\left(\frac{2 \mu_{\bar{k}_{h}}}{\mu_{\bar{k}_{h}}+\mu_{\bar{k}_{h}^{\prime}}}\right)^{\chi} \exp \left(\mu_{\ln W}+\frac{1}{2} \sigma_{\ln W}^{2}\right)$

778 where $\chi=\frac{v_{\bar{k}_{h}^{\prime}}}{v_{\bar{k}_{h}}}$ if $v_{\bar{k}_{h}}>v_{\bar{k}_{h}^{\prime}}$ and $\chi=\frac{v_{\bar{k}_{h}^{\prime}}-v_{\bar{k}_{h}}}{v_{\bar{k}_{h}^{\prime}}}$ if $v_{\bar{k}_{h}^{\prime}}>v_{\bar{k}_{h}}$ $m_{v}$ and $m_{v}^{\prime}$, respectively. of lognormally distributed $W$ with reasonable accuracy as follows:

$779 \quad \sigma_{W}^{2}=\left(\frac{2 v_{\bar{k}_{h}^{\prime}}}{v_{\bar{k}_{h}}+v_{\bar{k}_{h}^{\prime}}}\right) \mu_{W}^{2}\left[\exp \left(\sigma_{\ln W}^{2}\right)-1\right]$

where $\gamma\left(D_{u}\right)_{k_{h}}, \gamma\left(D_{s}\right)_{k_{h}^{\prime}}, \gamma\left(D_{u}\right)_{m_{v}}$ and $\gamma\left(D_{s}\right)_{m_{v}^{\prime}}$ are the variance reduction factors for $k_{h}, k_{h}^{\prime}$,

It can be noticed that both $\mu_{\ln W}$ and $\sigma_{\ln W}^{2}$ of underlying normally distributed $\ln W$ are now

known. So $\mu_{W}$ and $\sigma_{W}^{2}$ of lognormally distributed $W$ can readily be obtained with reference to Eqs. (4) and (5). However, as $k_{h}$ and $k_{h}^{\prime}$ are not distributed over the entire soil domain and do not have the same influence on the overall behaviour of soil consolidation, the true $\mu_{W}$ and $\sigma_{W}^{2}$ will be somewhat different from those calculated directly using $\mu_{\ln W}$ and $\sigma_{\ln W}^{2}$. For this reason, the expressions for $\mu_{W}$ and $\sigma_{W}^{2}$ are empirically adjusted to obtain these two parameters 


$$
\mu_{V}=\left(\frac{2 \mu_{\bar{m}_{v}}}{\mu_{\bar{m}_{v}}+\mu_{\bar{m}_{v}^{\prime}}}\right)^{\gamma} \exp \left(\mu_{\ln V}+\frac{1}{2} \sigma_{\ln V}^{2}\right)
$$

783 where $\gamma=\frac{v_{\bar{m}_{v}^{\prime}}}{v_{\bar{m}_{v}}}$ if $v_{\bar{m}_{v}}>v_{\bar{m}_{v}^{\prime}}$ and $\gamma=\frac{v_{\bar{m}_{v}^{\prime}}-v_{\bar{m}_{v}}}{v_{\bar{m}_{v}^{\prime}}}$ if $v_{\bar{m}_{v}^{\prime}}>v_{\bar{m}_{v}}$

784

$$
\sigma_{V}^{2}=\left(\frac{2 v_{\bar{m}_{v}^{\prime}}}{v_{\bar{m}_{v}}+v_{\bar{m}_{v}^{\prime}}}\right) \mu_{V}^{2}\left[\exp \left(\sigma_{\ln V}^{2}\right)-1\right]
$$

In Eqs. (B.11-B.14), $v_{\bar{k}_{h}}=\sigma_{\bar{k}_{h}} / \mu_{\bar{k}_{h}}$ and $v_{\bar{k}_{h}^{\prime}}=\sigma_{\bar{k}_{h}^{\prime}} / \mu_{\bar{k}_{h}^{\prime}}$ are the coefficients of variation of the equivalent permeability in the undisturbed and smear zones, respectively $\left(\sigma_{\bar{k}_{h}}, \mu_{\bar{k}_{h}}, \sigma_{\bar{k}_{h}^{\prime}}\right.$ and $\mu_{\bar{k}_{h}^{\prime}}$ are the standard deviation and mean of $\bar{k}_{h}$ and $\bar{k}_{h}^{\prime}$, respectively); $v_{\bar{m}_{v}}=\sigma_{\bar{m}_{v}} / \mu_{\bar{m}_{v}}$ and 788 $v_{\bar{m}_{v}^{\prime}}=\sigma_{\bar{m}_{v}^{\prime}} / \mu_{\bar{m}_{v}^{\prime}}$ are the coefficients of variation of the equivalent volume compressibility in the undisturbed and smear zones, respectively $\left(\sigma_{\bar{m}_{v}}, \mu_{\bar{m}_{v}}, \sigma_{\bar{m}_{v}^{\prime}}\right.$ and $\mu_{\bar{m}_{v}^{\prime}}$ are the standard deviation and mean of $\bar{m}_{v}$ and $\bar{m}_{v}^{\prime}$, respectively). With reference to Eqs. (4) and (5), and making use of Eqs. (19) and (21) lead to the following equations of the mean and standard deviation of $\bar{k}_{h}$ and $\bar{k}_{h}^{\prime}$ :

$\mu_{\bar{k}_{h}}=\exp \left(\mu_{\ln \bar{k}_{h}}+\frac{1}{2} \sigma_{\ln \bar{k}_{h}}^{2}\right)=\exp \left(\ln \mu_{k_{h}}-\frac{1}{2} \ln \left(1+v_{k_{h}}^{2}\right)+\frac{1}{2} \gamma\left(D_{u}\right)_{k_{h}} \ln \left(1+v_{k_{h}}^{2}\right)\right)$

$\sigma_{\bar{k}_{h}}=\mu_{\bar{k}_{h}} \sqrt{\left[\exp \left(\sigma_{\ln \bar{k}_{h}}^{2}\right)-1\right]}=\mu_{\bar{k}_{h}} \sqrt{\left[\exp \left(\gamma\left(D_{u}\right)_{k_{h}} \ln \left(1+v_{k_{h}}^{2}\right)\right)-1\right]}$

$\mu_{\bar{k}_{h}^{\prime}}=\exp \left(\mu_{\ln \bar{k}_{h}^{\prime}}+\frac{1}{2} \sigma_{\ln \bar{k}_{h}^{\prime}}^{2}\right)=\exp \left(\ln \mu_{k_{h}^{\prime}}-\frac{1}{2} \ln \left(1+v_{k_{h}^{\prime}}^{2}\right)+\frac{1}{2} \gamma\left(D_{s}\right)_{k_{h}^{\prime}} \ln \left(1+v_{k_{h}^{\prime}}^{2}\right)\right)$

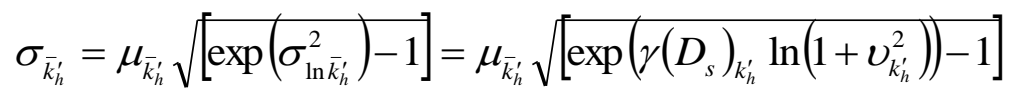

Similarly, the mean and standard deviation of $\bar{m}_{v}$ and $\bar{m}_{v}^{\prime}$ are thus: 
$\mu_{\bar{m}_{v}}=\exp \left(\ln \mu_{m_{v}}-\frac{1}{2} \ln \left(1+v_{m_{v}}^{2}\right)+\frac{1}{2} \gamma\left(D_{u}\right)_{m_{v}} \ln \left(1+v_{m_{v}}^{2}\right)\right)$

799

$\sigma_{\bar{m}_{v}}=\mu_{\bar{m}_{v}} \sqrt{\left[\exp \left(\gamma\left(D_{u}\right)_{m_{v}} \ln \left(1+v_{m_{v}}^{2}\right)\right)-1\right]}$

800

$\mu_{\bar{m}_{v}^{\prime}}=\exp \left(\ln \mu_{m_{v}^{\prime}}-\frac{1}{2} \ln \left(1+v_{m_{v}^{\prime}}^{2}\right)+\frac{1}{2} \gamma\left(D_{s}\right)_{m_{v}^{\prime}} \ln \left(1+v_{m_{v}^{\prime}}^{2}\right)\right)$

801

$$
\sigma_{\bar{m}_{v}^{\prime}}=\mu_{\bar{m}_{v}^{\prime}} \sqrt{\left[\exp \left(\gamma\left(D_{s}\right)_{m_{v}^{\prime}} \ln \left(1+v_{m_{v}^{\prime}}^{2}\right)\right)-1\right]}
$$

802 Substituting Eqs. (B.11) and (B.12) into Eqs. (B.3) and (B.5) lead to the following equations 803 of $\mu_{\bar{\alpha}}$ and $\sigma_{\bar{\alpha}}^{2}$ :

804

$\mu_{\bar{\alpha}}=a+b\left(\frac{2 \mu_{\bar{k}_{h}}}{\mu_{\bar{k}_{h}}+\mu_{\bar{k}_{h}^{\prime}}}\right)^{\chi}\left(\exp \left(\mu_{\ln W}+\frac{1}{2} \sigma_{\ln W}^{2}\right)\right)$

$805 \quad \sigma_{\bar{\alpha}}^{2}=\left(\frac{2 v_{\bar{k}_{h}^{\prime}}}{v_{\bar{k}_{h}}+v_{\bar{k}_{h}^{\prime}}}\right) b^{2} \mu_{W}^{2}\left[\exp \left(\sigma_{\ln W}^{2}\right)-1\right]$

806 Again substituting Eqs. (B.13) and (B.14) into Eqs. (B.4) and (B.6) gives:

807

$\mu_{\bar{\alpha}_{m_{v}}}=g+h\left(\frac{2 \mu_{\bar{m}_{v}}}{\mu_{\bar{m}_{v}}+\mu_{\bar{m}_{v}^{\prime}}}\right)^{\gamma}\left(\exp \left(\mu_{\ln V}+\frac{1}{2} \sigma_{\ln V}^{2}\right)\right)$

808

$$
\sigma_{\bar{\alpha}_{m_{v}}}^{2}=\left(\frac{2 v_{\bar{m}_{v}^{\prime}}}{v_{\bar{m}_{v}}+v_{\bar{m}_{v}^{\prime}}}\right) h^{2} \mu_{V}^{2}\left[\exp \left(\sigma_{\ln V}^{2}\right)-1\right]
$$

809 Finally, the statistics (i.e., $\mu_{\ln \bar{\alpha}}, \sigma_{\ln \bar{\alpha}}^{2}, \mu_{\ln \bar{\alpha}_{m_{v}}}$ and $\sigma_{\ln \bar{\alpha}_{m_{v}}}^{2}$ ) of the underlying normally

810 distributed $\ln \bar{\alpha}$ and $\ln \bar{\alpha}_{m_{v}}$ can be obtained from the obtained values of $\mu_{\bar{\alpha}}, \sigma_{\bar{\alpha}}^{2}, \mu_{\bar{\alpha}_{m_{v}}}$ and $811 \sigma_{\bar{\alpha}_{m_{v}}}^{2}$ by using Eqs. (2) and (3).

812 All six requested parameters (i.e., $\mu_{\ln \bar{k}_{h}}, \mu_{\ln \bar{m}_{v}}, \mu_{\ln \bar{\alpha}}, \mu_{\ln \bar{\alpha}_{m_{v}}}, \sigma_{\ln \bar{\alpha}}^{2}$ and $\sigma_{\ln \bar{\alpha}_{m_{v}}}^{2}$ ) are now 813 known and can be used in Eqs. (42) and (43) for the estimation of $\mu_{\ln U^{*}(t)}$ and $\sigma_{\ln U^{*}(t)}^{2}$. Using 
the developed semi-analytical relationships, the procedure for calculating $\mu_{\ln U^{*}(t)}$ and $\sigma_{\ln U^{*}(t)}^{2}$ can be summarized as follows:

1. Determine all constant parameters involved in the RBSA-G2C1 (i.e., $C, a, b, g, h$, $\gamma\left(D_{u}\right)_{k_{h}}, \gamma\left(D_{s}\right)_{k_{h}^{\prime}}, \gamma\left(D_{u}\right)_{m_{v}}$ and $\left.\gamma\left(D_{s}\right)_{m_{v}^{\prime}}\right)$

2. Calculate $\mu_{\ln \bar{k}_{h}}$ and $\mu_{\ln \bar{m}_{v}}$ from Eqs. (B.1) and (B.2);

3. Calculate $\mu_{\ln W}, \sigma_{\ln W}^{2}, \mu_{\ln V}$ and $\sigma_{\ln V}^{2}$ using Eqs. (B.7)-(B.10);

4. Calculate $\mu_{\bar{k}_{h}}, \sigma_{\bar{k}_{h}}, \mu_{\bar{k}_{h}^{\prime}}, \sigma_{\bar{k}_{h}^{\prime}}, \mu_{\bar{m}_{v}}, \sigma_{\bar{m}_{v}}, \mu_{\bar{m}_{v}^{\prime}}$ and $\sigma_{\bar{m}_{v}^{\prime}}$ using Eqs. (B.15)-(B.22), then determine $v_{\bar{k}_{h}}, v_{\bar{k}_{h}^{\prime}}, v_{\bar{m}_{v}}$ and $v_{\bar{m}_{v}^{\prime}}$

5. Calculate $\mu_{W}$ and $\mu_{V}$ using Eqs. (B.11) and (B.13);

6. Using the values of $\mu_{\ln W}, \sigma_{\ln W}^{2}, \mu_{W}, \mu_{\ln V}, \sigma_{\ln V}^{2}, \mu_{V}, v_{\bar{k}_{h}}, v_{\bar{k}_{h}^{\prime}}, v_{\bar{m}_{v}}$ and $v_{\bar{m}_{v}^{\prime}}$ obtained in Steps 3-5, calculate $\mu_{\bar{\alpha}}, \sigma_{\bar{\alpha}}^{2}, \mu_{\bar{\alpha}_{m_{\nu}}}$ and $\sigma_{\bar{\alpha}_{m_{\nu}}}^{2}$ from Eqs. (B.23)-(B.26);

7. Use Eqs. (2) and (3) to determine $\sigma_{\ln \bar{\alpha}}^{2}, \mu_{\ln \bar{\alpha}}, \sigma_{\ln \bar{\alpha}_{m_{v}}}^{2}$ and $\mu_{\ln \bar{\alpha}_{m_{v}}}$ from the obtained values of $\mu_{\bar{\alpha}}, \sigma_{\bar{\alpha}}^{2}, \mu_{\bar{\alpha}_{m_{v}}}$ and $\sigma_{\bar{\alpha}_{m_{v}}}^{2}$ in Step 6; and

8. Evaluate $\mu_{\ln U^{*}(t)}$ and $\sigma_{\ln U^{*}(t)}^{2}$ by substituting $\mu_{\ln \bar{k}_{h}}, \mu_{\ln \bar{m}_{v}}, \mu_{\ln \bar{\alpha}}$ and $\mu_{\ln \bar{\alpha}_{m_{v}}}$ in Eq. (42), and $\sigma_{\ln \bar{\alpha}}^{2}$ and $\sigma_{\ln \bar{\alpha}_{m_{v}}}^{2}$ in Eq. (43).

\section{Appendix C. RBSA model considering permeability as the only random variable}

G1C2: RBSA model considering $k_{h}$ as continuous random variables over the entire unit cell and $m_{v}$ deterministic

The spatial variability of $m_{v}$ is generally much less than that of $k_{h}$. Therefore, it is not unlikely to encounter soil with no or very little variability in $m_{v}$. For such condition, $m_{v}$ can be 
838

$$
C=\frac{2 t}{r_{e}^{2} m_{v} \gamma_{w} \alpha \alpha_{m_{v}}}
$$

839 The expressions for $\mu_{\ln U^{*}(t)}$ and $\sigma_{\ln U^{*}(t)}^{2}$ given in Eqs. (33) and (34) are then reduced to:

840

841

842

848

$$
C=\frac{2 t}{r_{e}^{2} \gamma_{w} m_{v} \alpha_{m_{v}}}
$$

849 The equations for $\mu_{\ln U^{*}(t)}$ and $\sigma_{\ln U^{*}(t)}^{2}$ given in Eqs. (42) and (43) are then reduced to:

850

851

$$
\sigma_{\ln U^{*}(t)}^{2}=\sigma_{\ln \bar{\alpha}}^{2}
$$

852 The three unknown parameters: $\mu_{\ln \bar{k}_{h}}, \mu_{\ln \bar{\alpha}}$ and $\sigma_{\ln \bar{\alpha}}^{2}$ in Eqs. (C.5) and (C.6) are already

853 determined during the course of the development of the RBSA-G2C1 model as presented in

854 Appendix B and can be readily used for estimation of $\mu_{\ln U^{*}(t)}$ and $\sigma_{\ln U^{*}(t)}^{2}$. 


\section{References}

858

859

[1] Barron RA. Consolidation of fine-grained soils by drain wells. Transactions of the

860

861

862

863

864

865

866

867

868

869

870

871

872

873

874

875

876

877

878

879

880

881

American Society of Civil Engineering 1948;113(718-54.

[2] Hansbo S. Consolidation of fine-grained soils by prefabricated drains. Proceedings of the 10th International Conference on Soil Mechanics and Foundation Engineering. Stockholm, Sweden; 1981. p. 677-82.

[3] Onoue A. Consolidation by vertical drains taking well resistance and smear into consideration. Soils and Foundations 1988;24(4):165-74.

[4] Chang CS. Uncertainty of one-dimensional consolidation analysis. Journal of Geotechnical Engineering 1985;111(12):1411-24.

[5] Freeze RA. Probabilistic one-dimensional consolidation. Journal of Geotechnical Engineering Division 1977;103(GT7):725-42.

[6] Huang J, Griffiths DV, Fenton GA. Probabilistic analysis of coupled soil consolidation. Journal of Geotechnical and Geoenvironmental Engineering 2010;136(3):417-30.

[7] Hong HP, Shang JQ. Probabilistic analysis of consolidation with prefabricated vertical drains for soil improvement. Canadian Geotechnical Journal 1998;35(4):666-77.

[8] Zhou W, Hong HP, Shang JQ. Probabilistic design method of prefabricated vertical drains for soil improvement. Journal of Geotechnical and Geoenvironmental Engineering 1999;125(8):659-64.

[9] Walker RT, Indraratna B. Vertical drain consolidation with parabolic distribution of permeability in smear zone. Journal of Geotechnical and Geoenvironmental Engineering 2006;132(7):937-41.

[10] Basu D, Basu P, Prezzi M. Analytical solutions for consolidation aided by vertical drains.

Geomechanics and Geoengineering: An International Journal 2006;1(1):63-71. 
882

883

884

885

886

887

888

889

890

891

892

893

894

895

896

897

898

899

900

901

902

903

904

905

906

[11] Fenton G, Griffiths DV. Probabilistic foundation settlement on spatially random soil. Journal of Geotechnical and Geoenvironmental Engineering 2002;128(5):381-90.

[12] Griffiths DV, Fenton GA. Probabilistic settlement analysis by stochastic and random finite-element methods. Journal of geotechnical and geoenvironmental engineering 2009;135(11):1629-37.

[13] Fenton GA, Vanmarcke EH. Simulation of random fields via local average subdivision. Journal of Engineering Mechanics 1990;116(8):1733-49.

[14] Pyrah IC. One-dimensional consolidation of layered soils. Geotechnique 1996;46(3):55560.

[15] Vanmarcke EH. Random fields: analysis and synthesis. Massachusetts: The MIT Press, 1984.

[16] Griffiths DV, Fenton GA. Seepage beneath water retaining structures founded on spatially random soil. Géotechnique 1993;43(4):577-87.

[17] Fenton GA, Griffiths DV. Risk assessment in geotechnical engineering. New Jersey: John Wiley and Sons, 2008.

[18] Lloret-Cabot M, Fenton GA, Hicks MA. On the estimation of scale of fluctuation in geostatistics. Georisk 2014;8(2):129-40.

[19] Smith IM, Griffiths DV. Programming the finite element method. 4th ed. Chichester, West Sussex: John Wiley and Sons, 2004.

[20] Ching J, Phoon K-K. Effect of element sizes in random field finite element simulations of soil shear strength. Computers \& structures 2013;126(1):120-34.

[21] Harada T, Shinozuka M. The scale of correlation for stochastic fields. New York: Department of Civil Engineering and Engineering Mechanics, Columbia University, 1986.

[22] Sharma JS, Xiao D. Characterization of a smear zone around vertical drains by largescale laboratory tests. Canadian Geotechical Journal 2000;37(6):1265-71. 
907 [23] Chu J, Bo MW, Choa V. Practical considerations for using vertical drains in soil 908 improvement projects. Geotextiles and Geomembranes 2004;22(1-2):101-17.

909 [24] Walker RT. Analytical solutions for modeling soft soil consolidation by vertical drains. 910 Wollongong, Australia: Thesis (PhD). University of Wollongong, 2006.

911 [25] Beacher GB, Christian JT. Reliability and Statistics in Geotechnical Engineering. 912 Chichester, England: John Wiley \& Sons, 2003.

913 [26] Phoon KK, Kulhawy FH. Characterisation of geotechnical variability. Canadian 914 Geotechnical Journal 1999;36(4):612-24.

915 [27] Bari MW, Shahin MA, Soubra A-H. Single vs multi-drain analyses of probabilistic soil 916 consolidation via prefabricated vertical drains. 12th International Conference on Applications 917 of Statistics and Probability in Civil Engineering. Vancouver, Canada; 2015 (accepted).

918 [28] Rixner JJ, Kraemer SR, Smith AD. Prefabricated vertical drains, Vol. I: Engineering 919 guidelines. Washington D. C.: Federal Highway Administration, 1986.

920 [29] Benjamin JR, Cornell CA. Probability, statistics, and decision for civil engineers. New 921 York: McGraw-Hill, 1970. 
932

933

934

935

936

937

938

939

940

941

942

943

944

945

946

947

948

949

950

951

952

953

954

\section{Figure Captions:}

Fig. 1. Schematic diagram of soil consolidation with prefabricated vertical drain: (a) cylindrical unit cell; (b) equivalent square geometry with FE mesh discretization

Fig. 2. Comparison between FEMC and RBSA-G1C1 for the effect of: (a) $v$ on $\mu_{\ln U^{*}}$ for $\theta=$ $0.5 \mathrm{~m}$ (b) $\theta$ on $\mu_{\ln U^{*}}$ for $v_{k_{h}}=200 \%, v_{m_{v}}=20 \%$ (c) $v$ on $\sigma_{\ln U^{*}}$ for $\theta=0.5 \mathrm{~m}$ and (d) $\theta$ on $\sigma_{\ln U^{*}}$ for $v_{k_{h}}=200 \%, v_{m_{v}}=20 \%$

Fig. 3. Comparison between FEMC and RBSA-G1C1 for the effect of: (a) $v$ on $P\left[U \geq U_{90}\right]$ for $\theta=0.5 \mathrm{~m}$ (b) $\theta$ on $P\left[U \geq U_{90}\right]$ for $v_{k_{h}}=200 \%, v_{m_{v}}=20 \%$

Fig. 4. Comparison between FEMC and RBSA-G2C1 for the effect of: (a) $v_{u}$ on $\mu_{\ln U^{*}}$ at fixed value of $v_{k_{h}^{\prime}}=100 \%, v_{m_{v}^{\prime}}=10 \%, \theta_{u}=\theta_{s}=1.0 \mathrm{~m}$; (b) $v_{s}$ on $\mu_{\ln U^{*}}$ at fixed value of $v_{k_{h}}=100 \%$, $v_{m_{v}}=10 \%, \theta_{u}=\theta_{s}=1.0 \mathrm{~m}$

Fig. 5. Comparison between FEMC and RBSA-G2C1 for the effect of: (a) $\theta_{u}$ on $\mu_{\ln U^{*}}$ at fixed value of $v_{k_{k}}=v_{k_{h}^{\prime}}=200 \%, v_{m_{v}}=v_{m_{v}^{\prime}}=20 \%, \theta_{s}=0.25 \mathrm{~m}$; (b) $\theta_{s}$ on $\mu_{\ln U^{*}}$ at fixed value of $v_{k_{k}}=$ $v_{k_{h}^{\prime}}=200 \%, v_{m_{v}}=v_{m_{v}^{\prime}}=20 \%, \theta_{u}=0.25 \mathrm{~m}$

Fig. 6. Comparison between FEMC and RBSA-G2C1 for the effect of: (a) $v_{u}$ on $\sigma_{\ln U^{*}}$ at fixed value of $v_{k_{h}^{\prime}}=100 \%, v_{m_{v}^{\prime}}=10 \%, \theta_{u}=\theta_{s}=1.0 \mathrm{~m}$; (b) $v_{s}$ on $\sigma_{\ln U^{*}}$ at fixed value of $v_{k_{h}}=100 \%$, $v_{m_{v}}=10 \%, \theta_{u}=\theta_{s}=1.0 \mathrm{~m}$

Fig. 7. Comparison between FEMC and RBSA-G2C1 for the effect of: (a) $\theta_{u}$ on $\sigma_{\ln U^{*}}$ at fixed value of $v_{k_{k}}=v_{k_{h}^{\prime}}=200 \%, v_{m_{v}}=v_{m_{v}^{\prime}}=20 \%, \theta_{s}=0.25 \mathrm{~m} ;$ (b) $\theta_{s}$ on $\sigma_{\ln U^{*}}$ at fixed value $v_{k_{k}}=v_{k_{h}^{\prime}}$ $=200 \%, v_{m_{v}}=v_{m_{v}^{\prime}}=20 \%, \theta_{u}=0.25 \mathrm{~m}$

Fig. 8. Comparison between FEMC and RBSA-G2C1 for the effect of: (a) $v_{u}$ on $P\left[U \geq U_{90}\right]$ at fixed value of $v_{k_{h}^{\prime}}=100 \%, v_{m_{v}^{\prime}}=10 \%, \theta_{u}=\theta_{s}=1.0 \mathrm{~m}$; (b) $v_{s}$ on $P\left[U \geq U_{90}\right]$ at fixed value of $v_{k_{h}}=100 \%, v_{m_{v}}=10 \%, \theta_{u}=\theta_{s}=1.0 \mathrm{~m}$ 
955 Fig. 9. Comparison between FEMC and RBSA-G2C1 for the effect of: (a) $\theta_{u}$ on $P\left[U \geq U_{90}\right]$ 956 at fixed value of $v_{k_{k}}=v_{k_{h}^{\prime}}=200 \%, v_{m_{v}}=v_{m_{v}^{\prime}}=20 \%, \theta_{s}=0.25 \mathrm{~m}$ (b) $\theta_{s}$ on $P\left[U \geq U_{90}\right]$ at fixed 957 value $v_{k_{k}}=v_{k_{h}^{\prime}}=200 \%, v_{m_{v}}=v_{m_{v}^{\prime}}=20 \%, \theta_{u}=0.25 \mathrm{~m}$

958 Fig. 10. Comparison between (a) $\mu_{\ln U^{*}}$ (b) $\sigma_{\ln U^{*}}$ and (c) $P\left[U \geq U_{90}\right]$ obtained from FEMC and 959 RBS A methods for a unit cell having $L / S=5$ and anisotropic $\theta$

960

961

962

963

964

965

966

967

968

969

970

971

972

973

974

975

976

977

978 


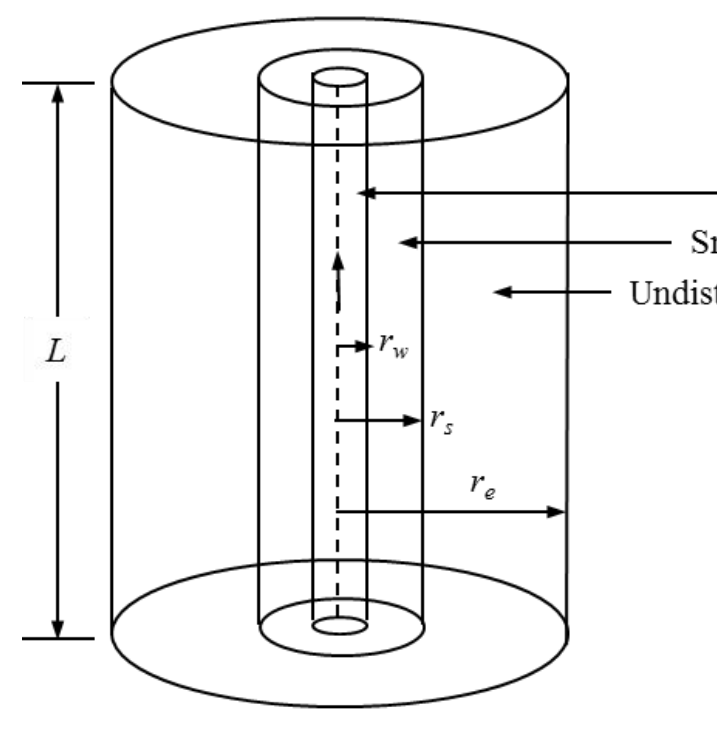

(a)

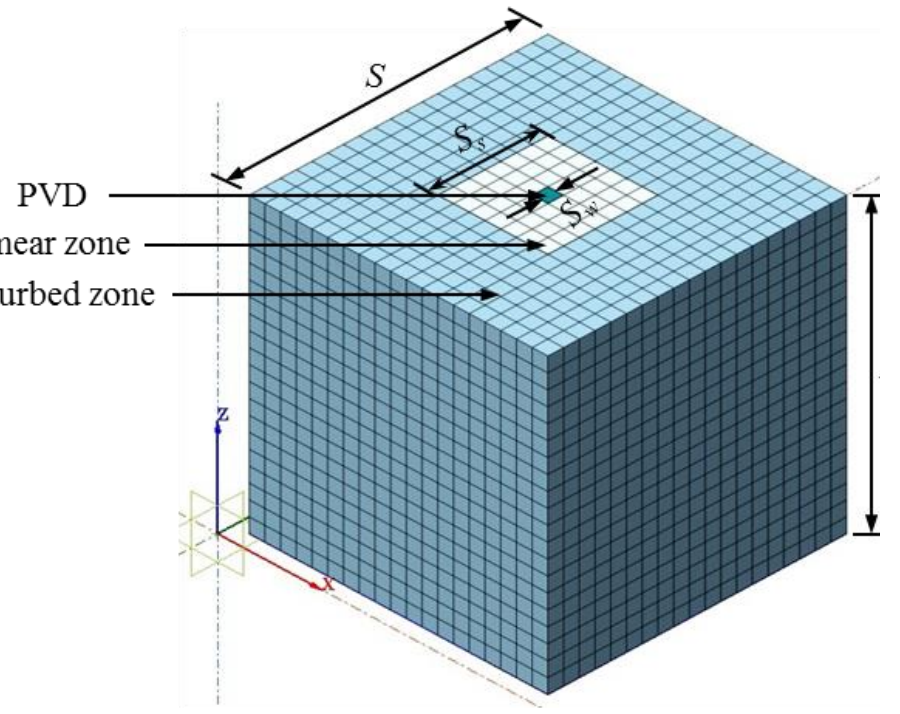

(b)

Fig. 1. Schematic diagram of soil consolidation with prefabricated vertical drain: (a) cylindrical unit cell; (b) equivalent square geometry with FE mesh discretization 

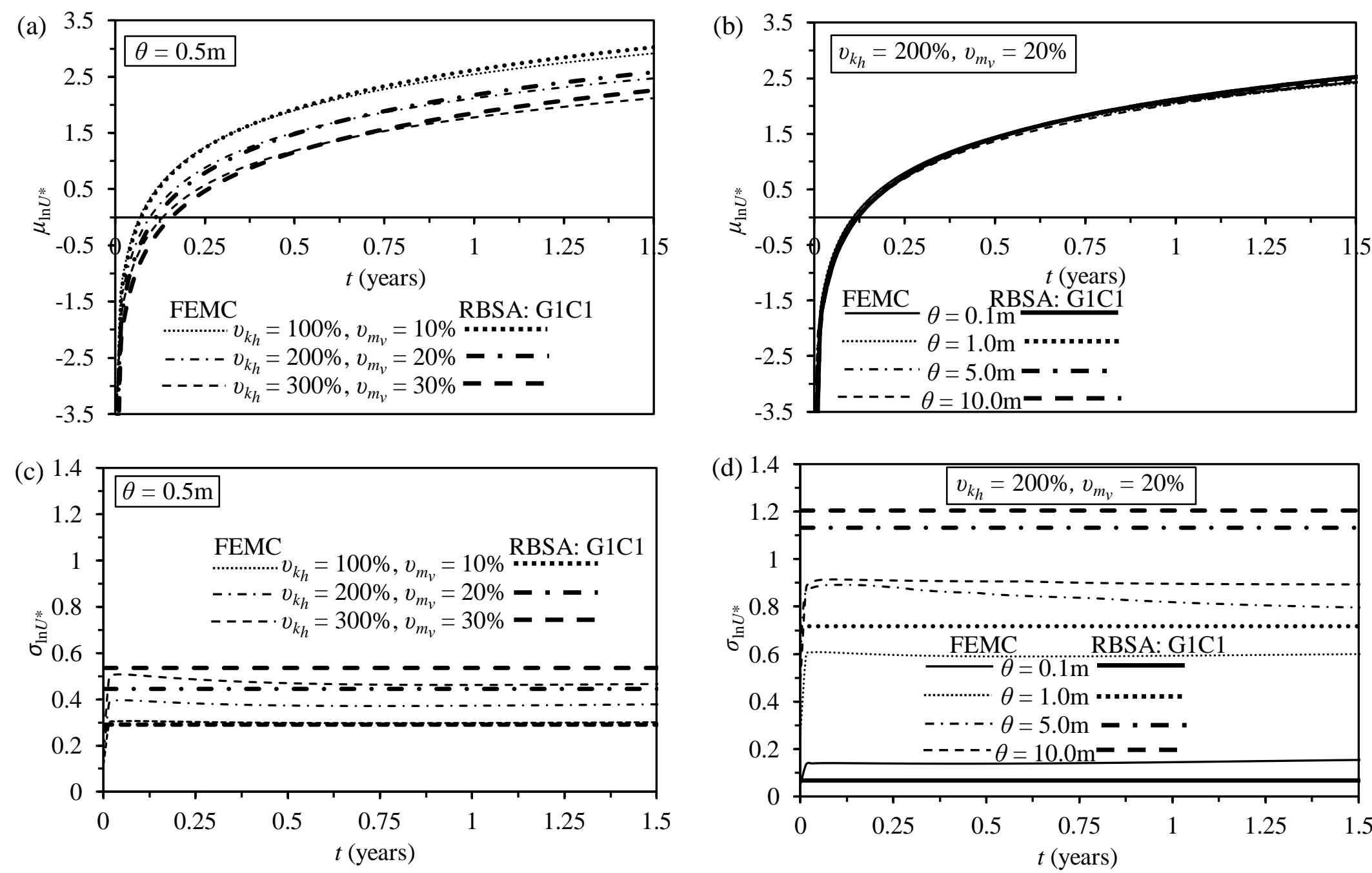

Fig. 2. Comparison between FEMC and RBSA-G1C1 for the effect of: (a) $v$ on $\mu_{\ln U^{*}}$ for $\theta=0.5 \mathrm{~m}$ (b) $\theta$ on $\mu_{\ln U^{*}}$ for $v_{k_{h}}=200 \%$, $v_{m_{v}}=20 \%$ (c) $v$ on $\sigma_{\ln U^{*}}$ for $\theta=0.5 \mathrm{~m}$ and (d) $\theta$ on $\sigma_{\ln U^{*}}$ for $v_{k_{h}}=200 \%, v_{m_{v}}=20 \%$ 

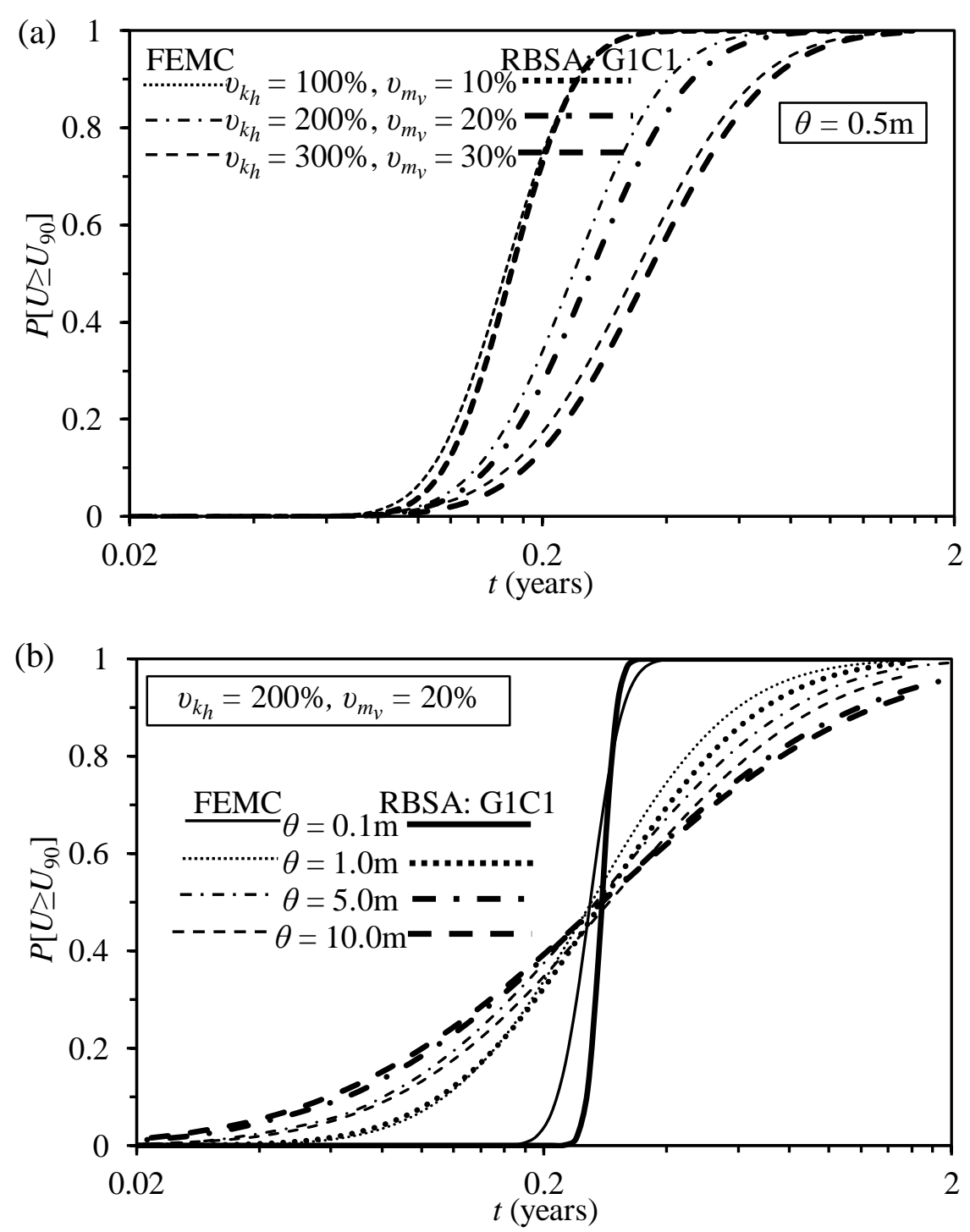

Fig. 3. Comparison between FEMC and RBSA-G1C1 for the effect of: (a) $v$ on $P\left[U \geq U_{90}\right]$ for $\theta$ $=0.5 \mathrm{~m}$ (b) $\theta$ on $P\left[U \geq U_{90}\right]$ for $v_{k_{h}}=200 \%, v_{m_{v}}=20 \%$ 

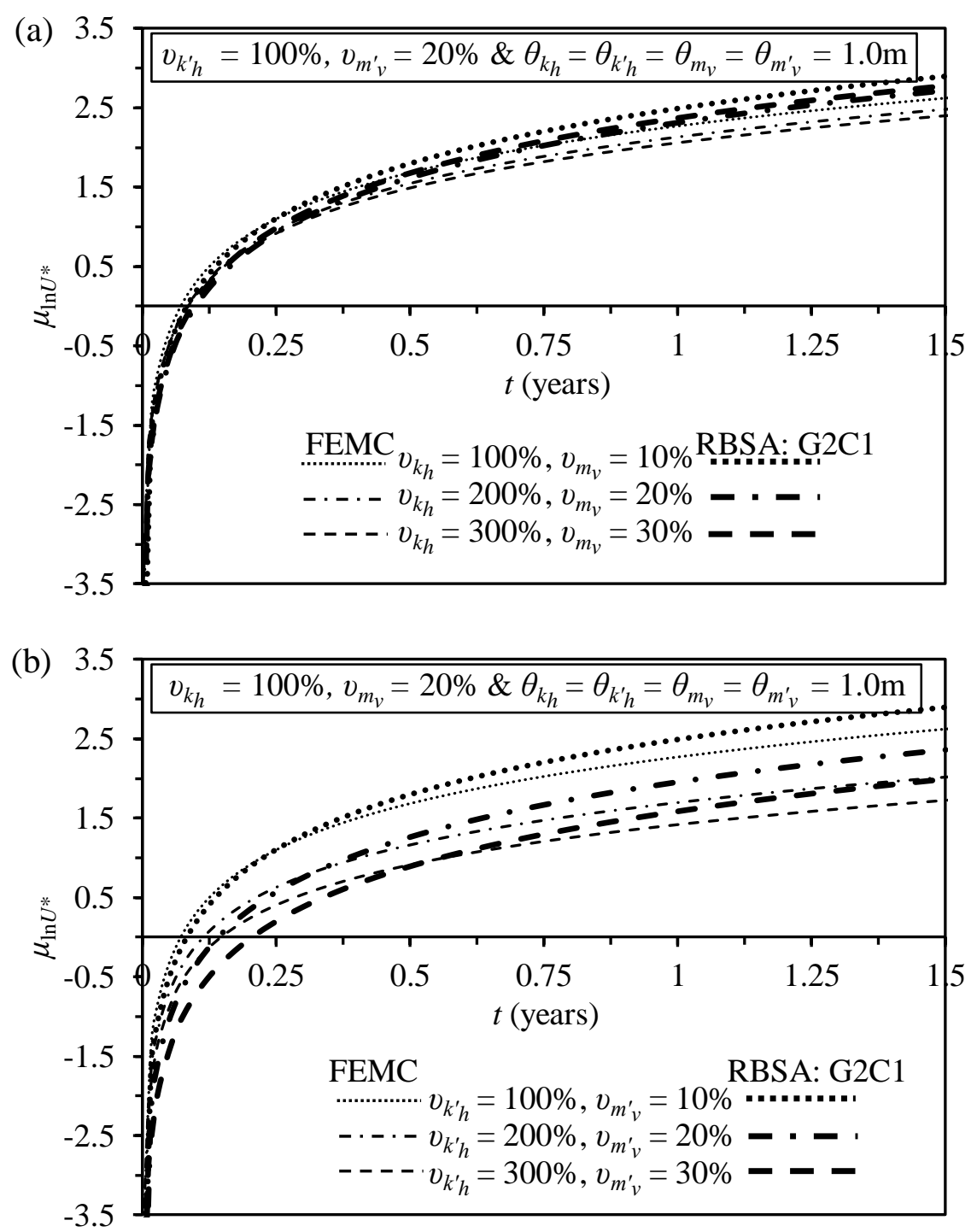

Fig. 4. Comparison between FEMC and RBSA-G2C1 for the effect of: (a) $v_{u}$ on $\mu_{\ln U^{*}}$ at fixed value of $v_{k_{h}^{\prime}}=100 \%, v_{m_{v}^{\prime}}=10 \%, \theta_{u}=\theta_{s}=1.0 \mathrm{~m}$; (b) $v_{s}$ on $\mu_{\ln U^{*}}$ at fixed value of $v_{k_{h}}=100 \%$,

$$
v_{m_{v}}=10 \%, \theta_{u}=\theta_{s}=1.0 \mathrm{~m}
$$



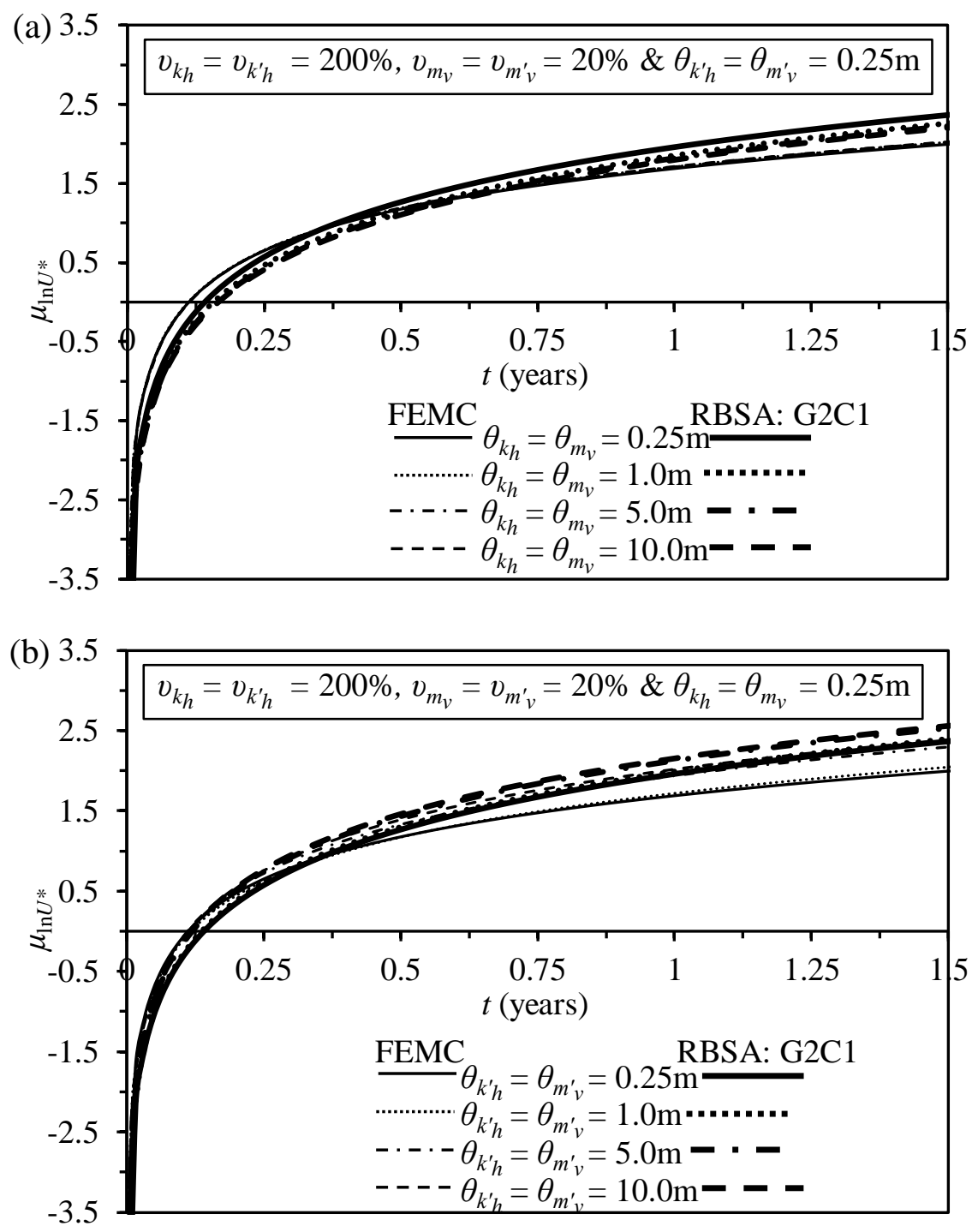

Fig. 5. Comparison between FEMC and RBSA-G2C1 for the effect of: (a) $\theta_{u}$ on $\mu_{\ln U^{*}}$ at fixed value of $v_{k_{k}}=v_{k_{h}^{\prime}}=200 \%, v_{m_{v}}=v_{m_{v}^{\prime}}=20 \%, \theta_{s}=0.25 \mathrm{~m}$; (b) $\theta_{s}$ on $\mu_{\ln U^{*}}$ at fixed value of $v_{k_{k}}=v_{k_{h}^{\prime}}$ $=200 \%, v_{m_{v}}=v_{m_{v}^{\prime}}=20 \%, \theta_{u}=0.25 \mathrm{~m}$ 

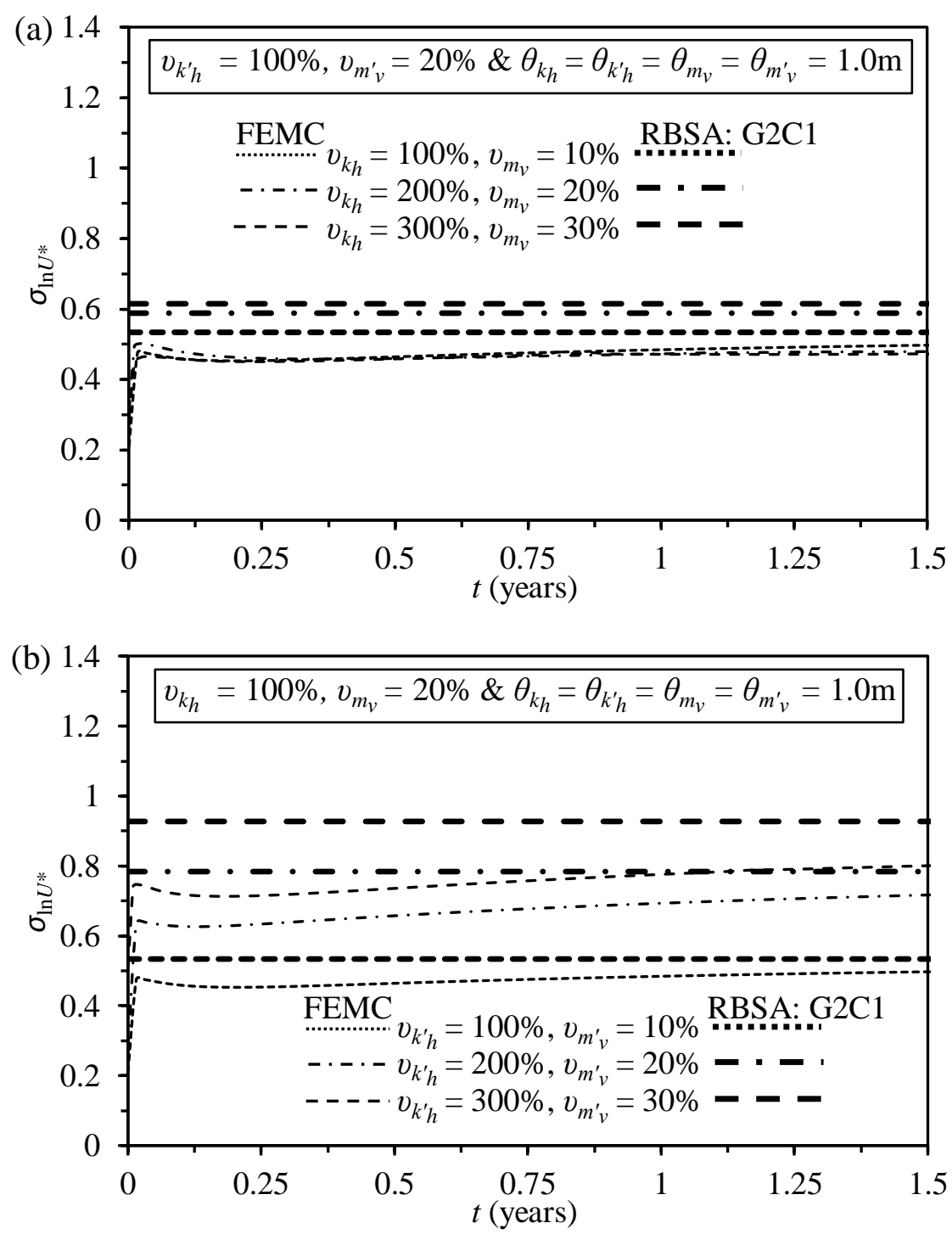

Fig. 6. Comparison between FEMC and RBSA-G2C1 for the effect of: (a) $v_{u}$ on $\sigma_{\ln U^{*}}$ at fixed value of $v_{k_{h}^{\prime}}=100 \%, v_{m_{v}^{\prime}}=10 \%, \theta_{u}=\theta_{s}=1.0 \mathrm{~m}$; (b) $v_{s}$ on $\sigma_{\ln U^{*}}$ at fixed value of $v_{k_{h}}=100 \%$,

$$
v_{m_{v}}=10 \%, \theta_{u}=\theta_{s}=1.0 \mathrm{~m}
$$



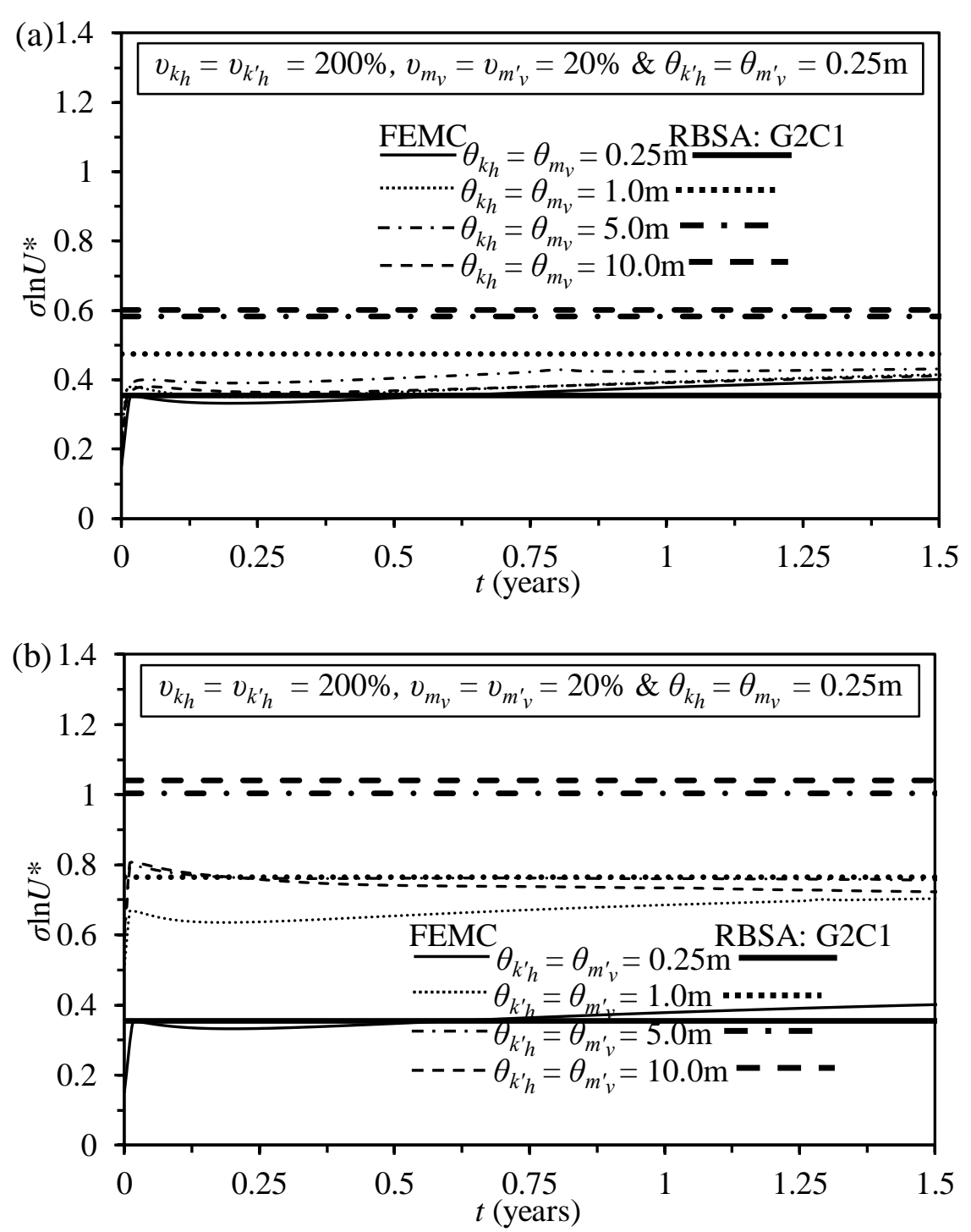

Fig. 7. Comparison between FEMC and RBSA-G2C1 for the effect of: (a) $\theta_{u}$ on $\sigma_{\ln U^{*}}$ at fixed value of $v_{k_{k}}=v_{k_{h}^{\prime}}=200 \%, v_{m_{v}}=v_{m_{v}^{\prime}}=20 \%, \theta_{s}=0.25 \mathrm{~m}$; (b) $\theta_{s}$ on $\sigma_{\ln U^{*}}$ at fixed value $v_{k_{k}}=v_{k_{h}^{\prime}}=$ $200 \%, v_{m_{v}}=v_{m_{v}^{\prime}}=20 \%, \theta_{u}=0.25 \mathrm{~m}$ 

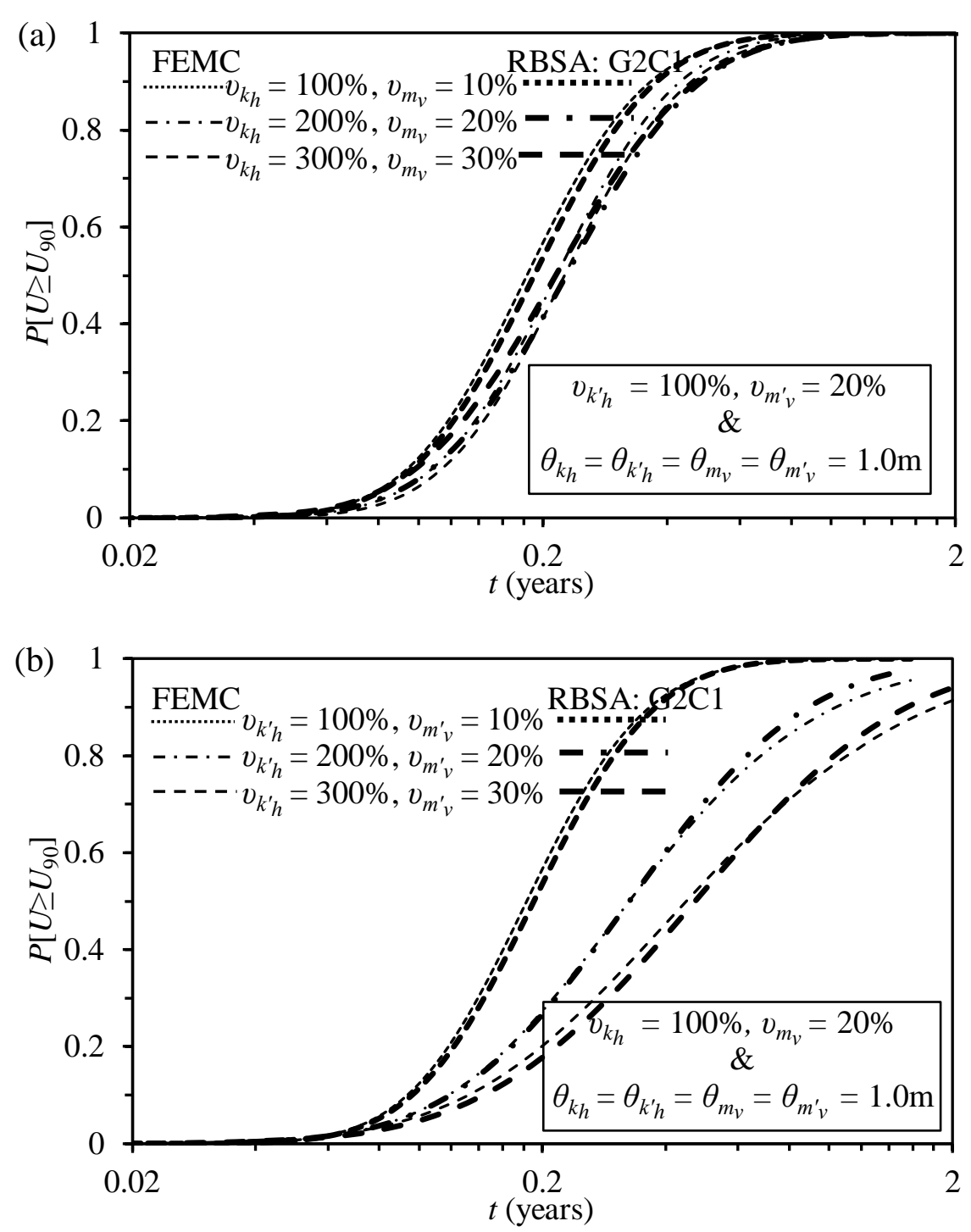

Fig. 8. Comparison between FEMC and RBSA-G2C1 for the effect of: (a) $v_{u}$ on $P\left[U \geq U_{90}\right]$ at fixed value of $v_{k_{h}^{\prime}}=100 \%, v_{m_{v}^{\prime}}=10 \%, \theta_{u}=\theta_{s}=1.0 \mathrm{~m}$; (b) $v_{s}$ on $P\left[U \geq U_{90}\right]$ at fixed value of $v_{k_{h}}$

$$
=100 \%, v_{m_{v}}=10 \%, \theta_{u}=\theta_{s}=1.0 \mathrm{~m}
$$



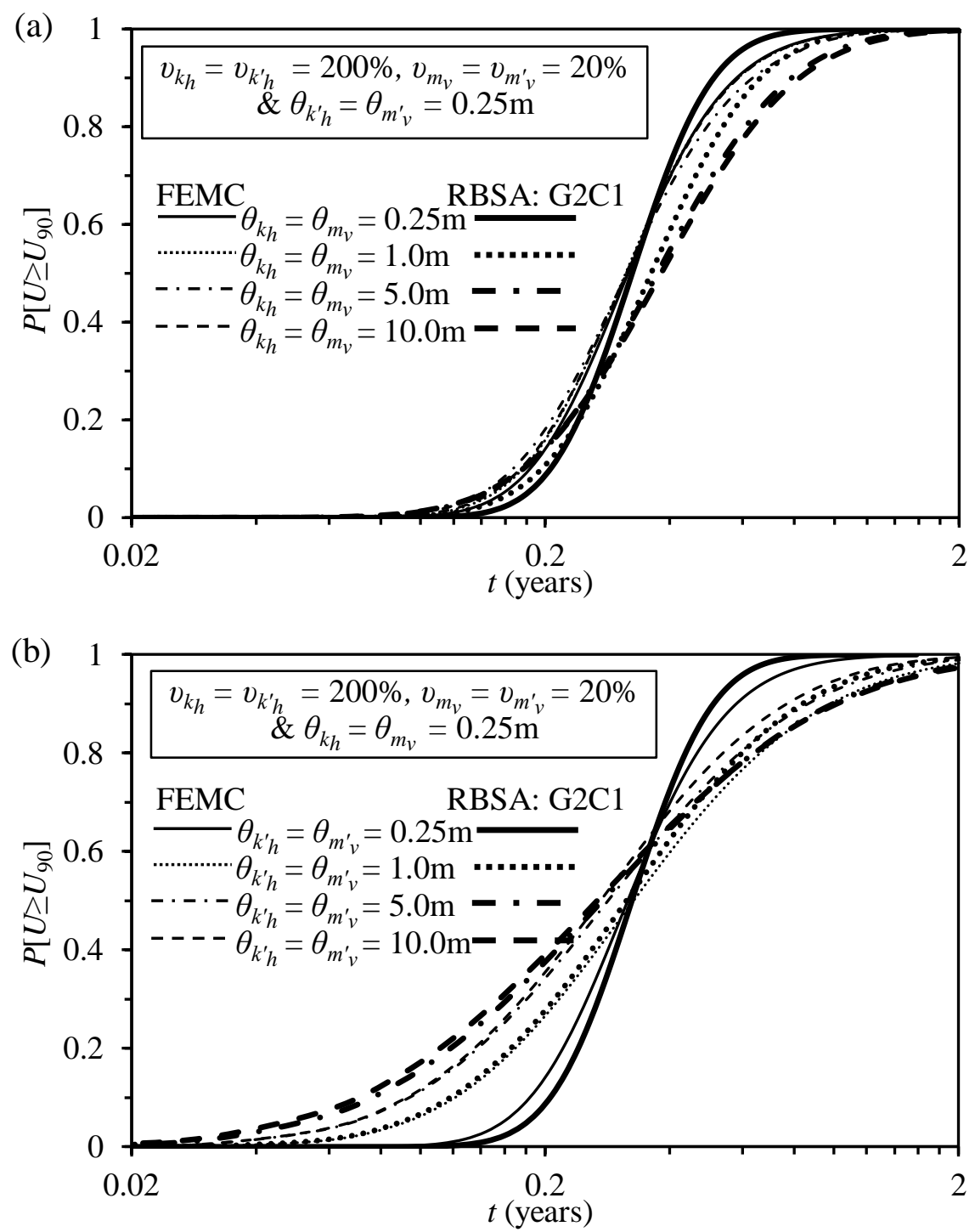

Fig. 9. Comparison between FEMC and RBSA-G2C1 for the effect of: (a) $\theta_{u}$ on $P\left[U \geq U_{90}\right]$ at fixed value of $v_{k_{k}}=v_{k_{h}^{\prime}}=200 \%, v_{m_{v}}=v_{m_{v}^{\prime}}=20 \%, \theta_{s}=0.25 \mathrm{~m}$ (b) $\theta_{s}$ on $P\left[U \geq U_{90}\right]$ at fixed value

$$
v_{k_{k}}=v_{k_{h}^{\prime}}=200 \%, v_{m_{v}}=v_{m_{v}^{\prime}}=20 \%, \theta_{u}=0.25 \mathrm{~m}
$$



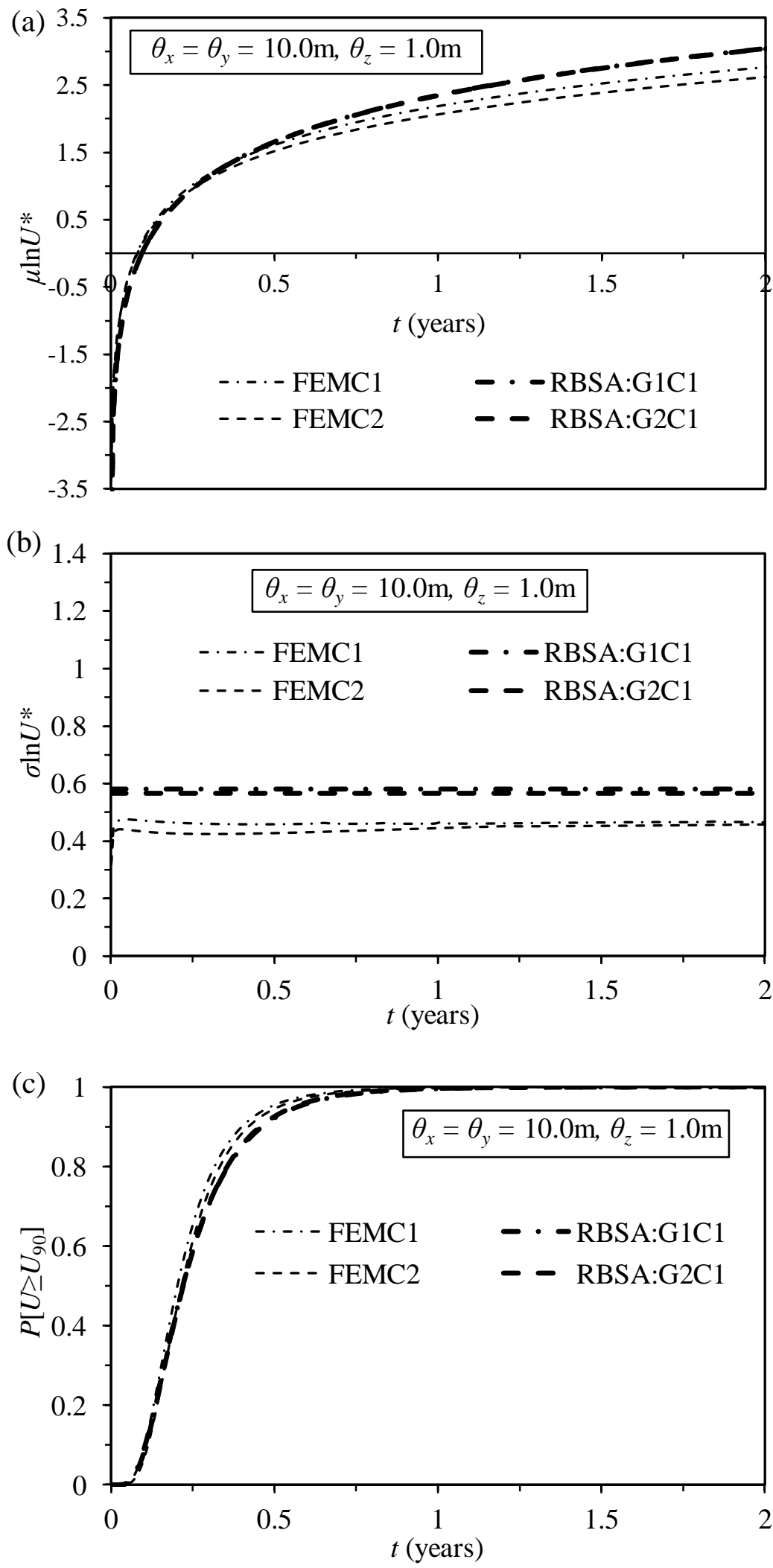

Fig. 10. Comparison between (a) $\mu_{\ln U^{*}}$ (b) $\sigma_{\ln U^{*}}$ and (c) $P\left[U \geq U_{90}\right]$ obtained from FEMC and RBSA methods for unit cell having $L / S=5$ and anisotropic $\theta$ 
